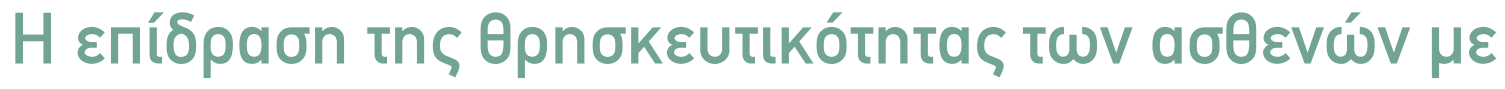

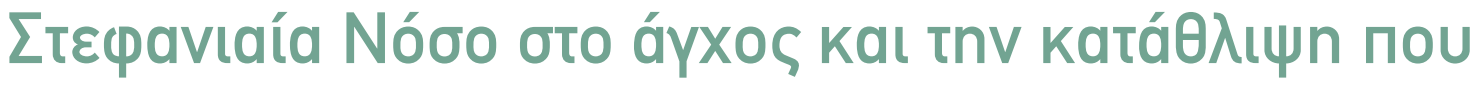

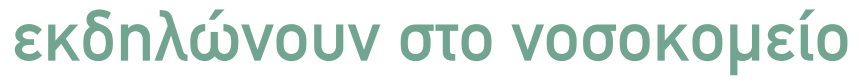

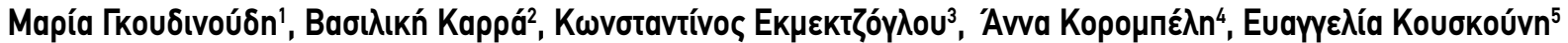

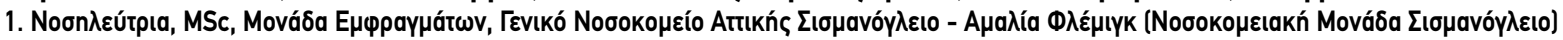

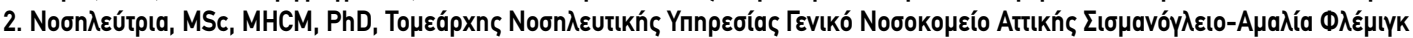

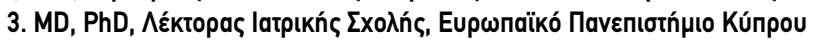

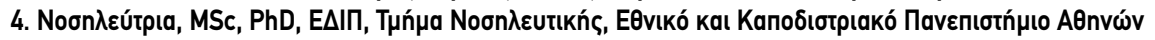

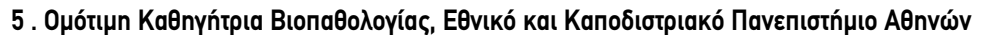

\section{ПEPIАHWH}

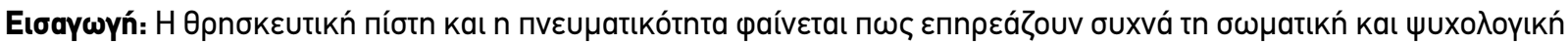

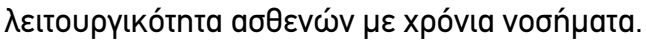

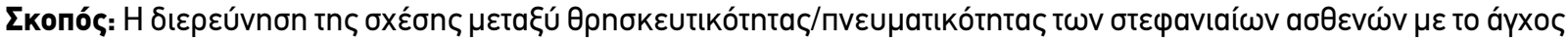

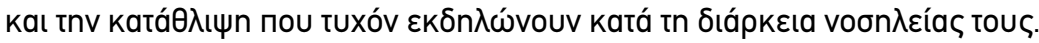

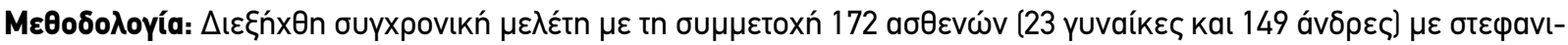

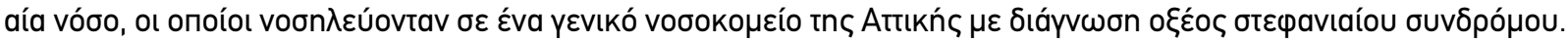

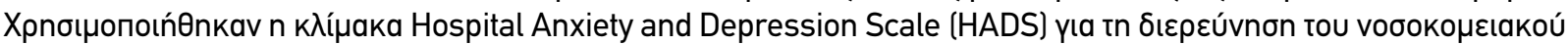

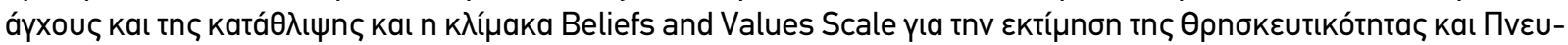

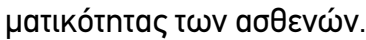

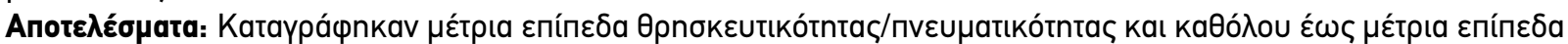

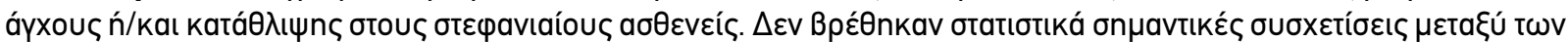

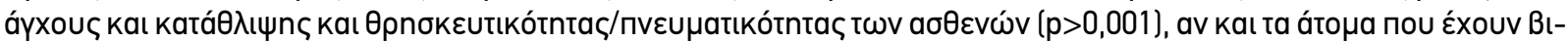

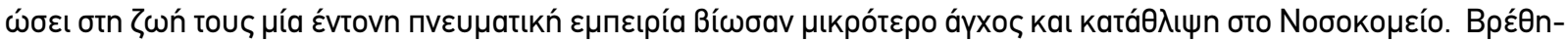

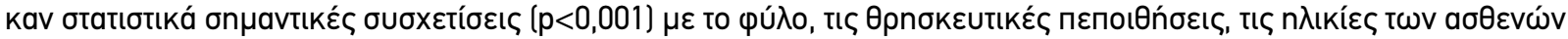

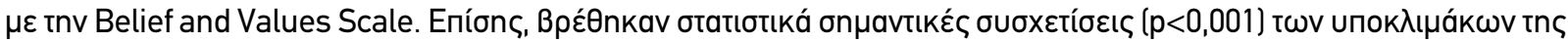

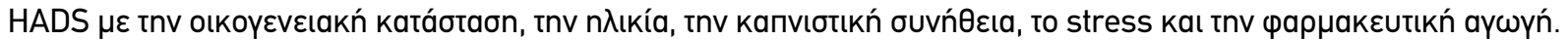

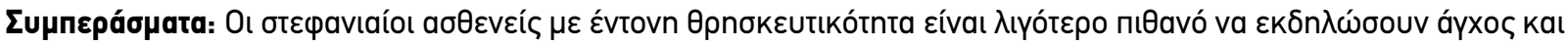

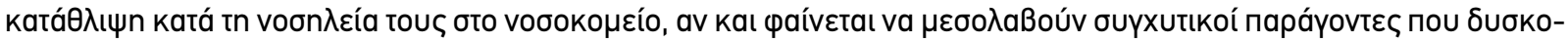

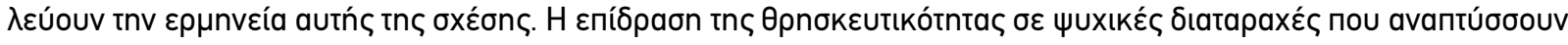

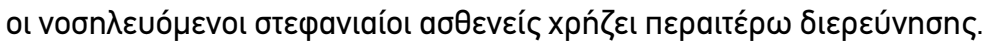

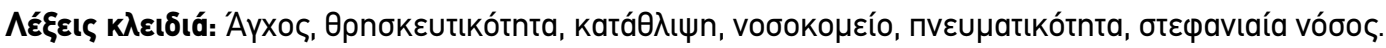




\section{EPEYNHTIKA APOPA}

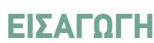

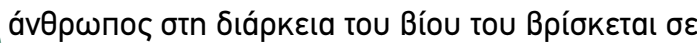

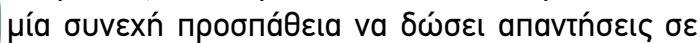

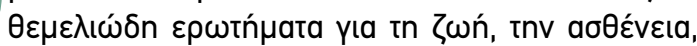

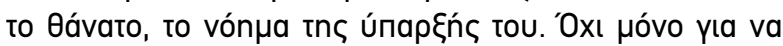

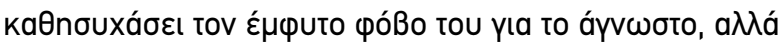

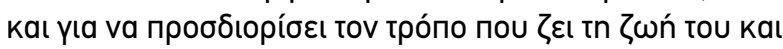

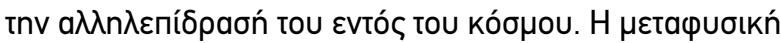

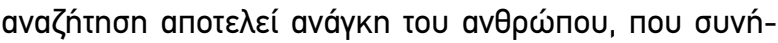

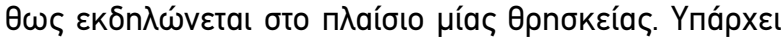

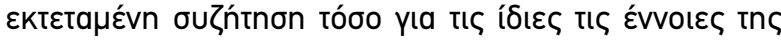

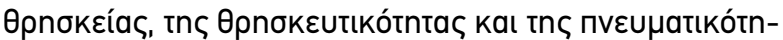

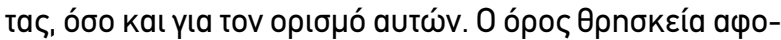

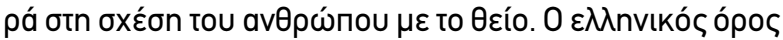

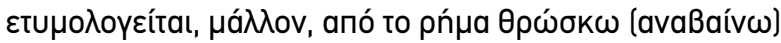

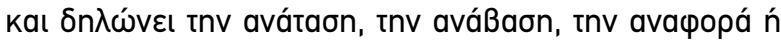

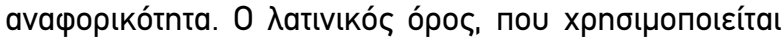

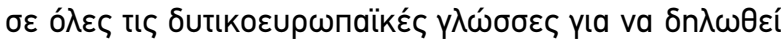

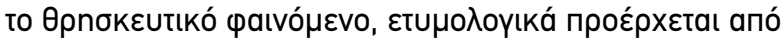

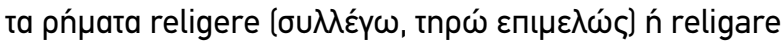

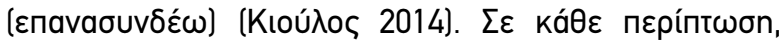

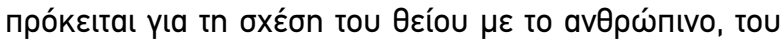

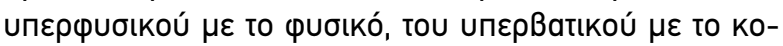

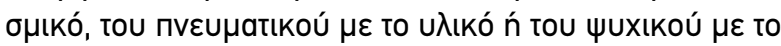

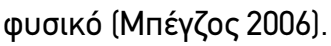

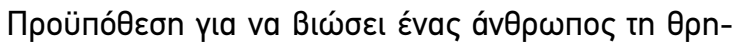

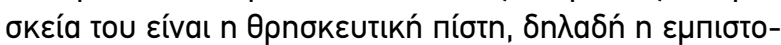

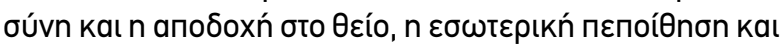

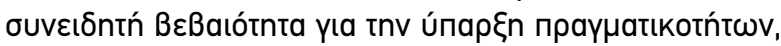

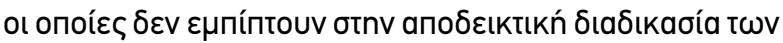

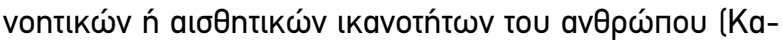

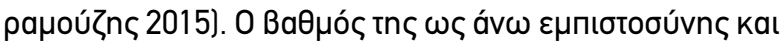

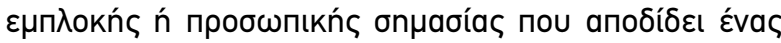

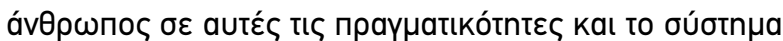

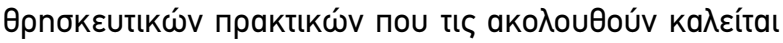

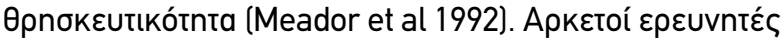

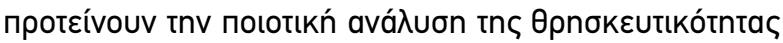

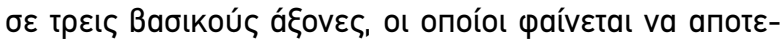

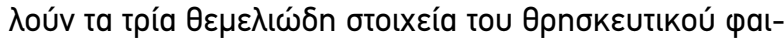

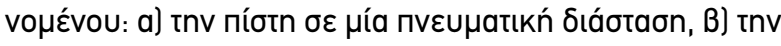

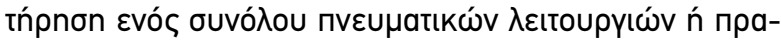

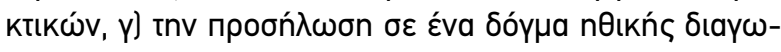

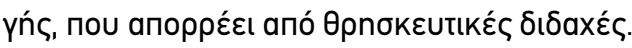

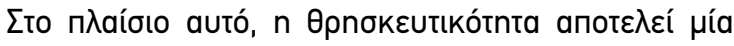

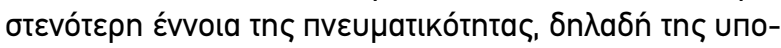

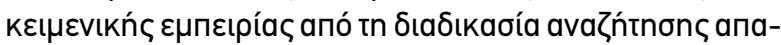

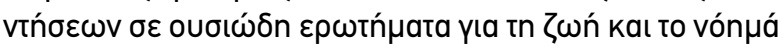

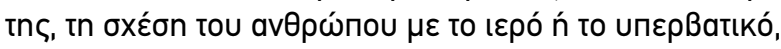

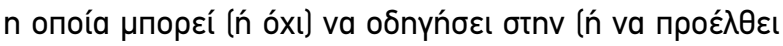

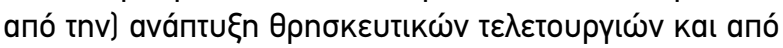

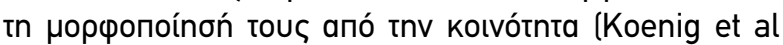

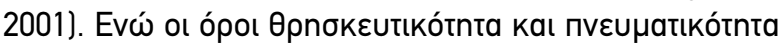

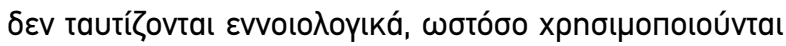

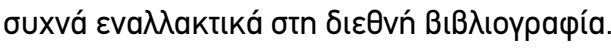

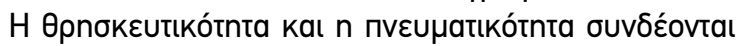

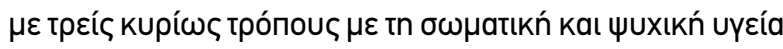

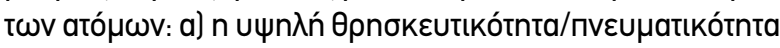

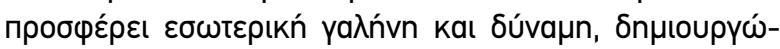

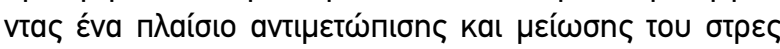

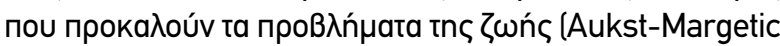

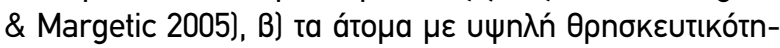

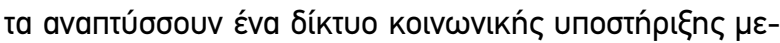

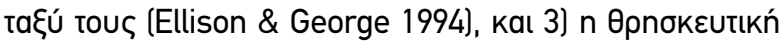

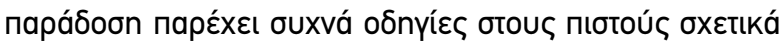

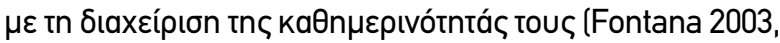

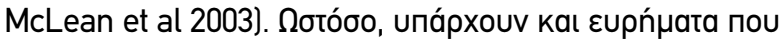

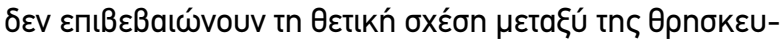

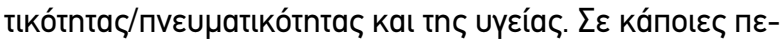

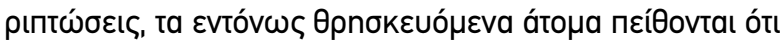

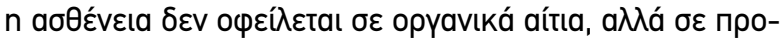

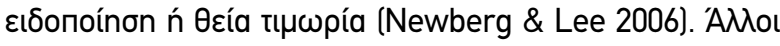

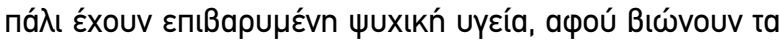

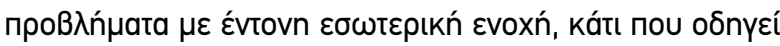

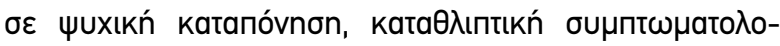

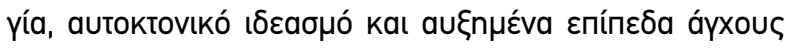
(Weber \& Pargament 2014).

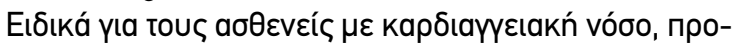

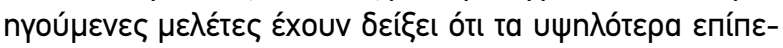

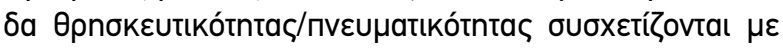

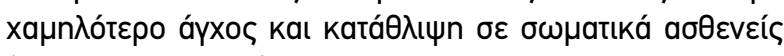

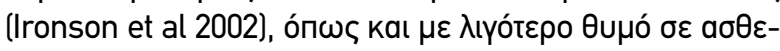

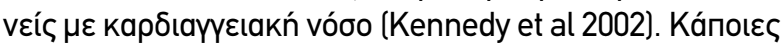

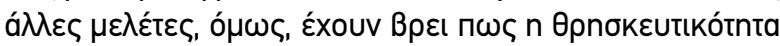

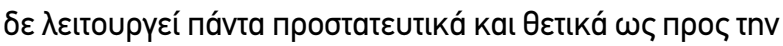

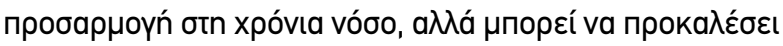

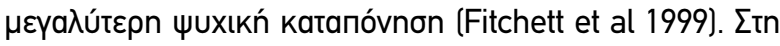

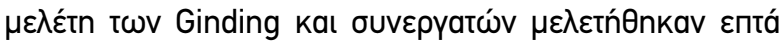

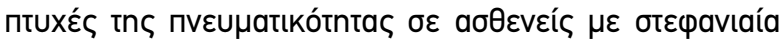

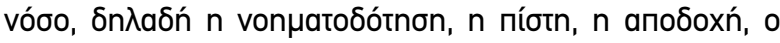

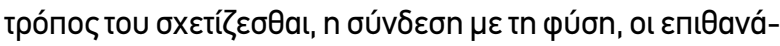

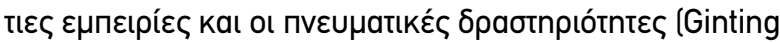

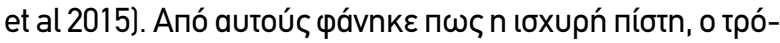

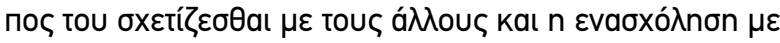

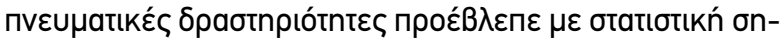

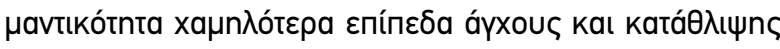

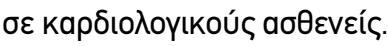

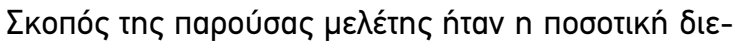

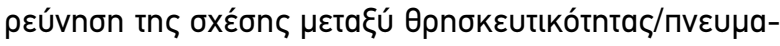

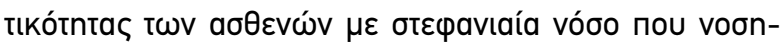

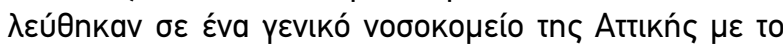
ápxoৎ kat tnv katá $\theta \lambda ı \psi n$ nou tuxóv $\varepsilon \kappa \delta n \lambda \omega ́ v o u v$ katá tn

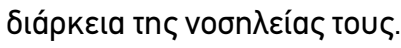

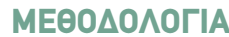

$\triangle \varepsilon$ íy

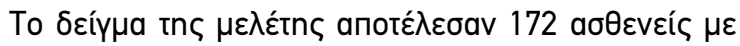




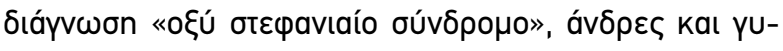

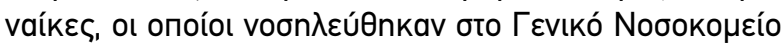

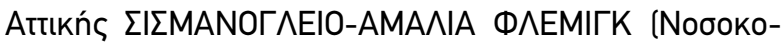

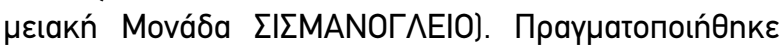

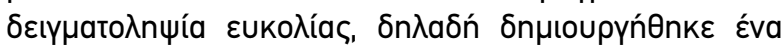

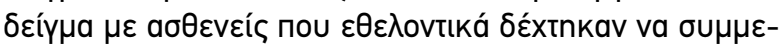

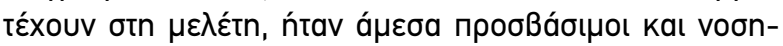

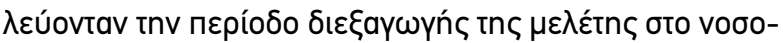

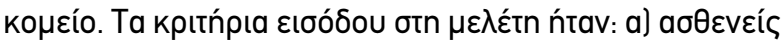

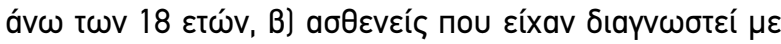

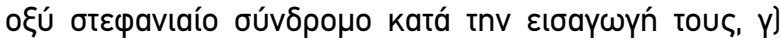

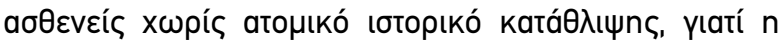

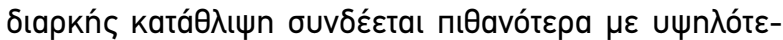

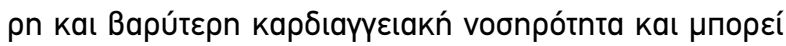

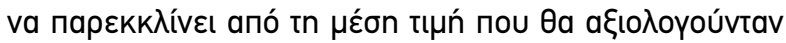

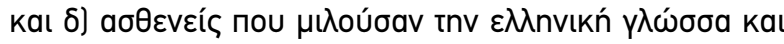

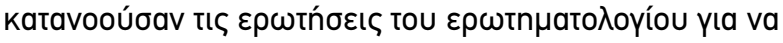

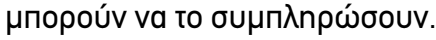

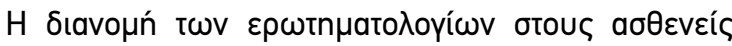

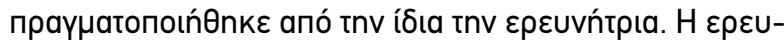

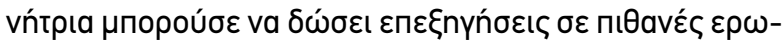

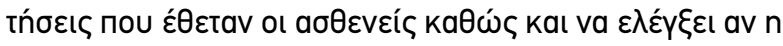

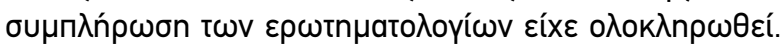

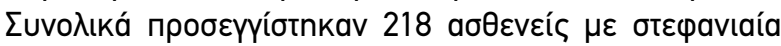

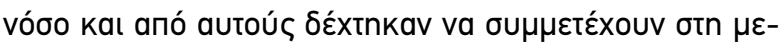

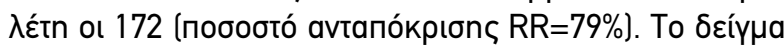

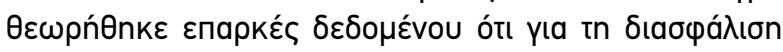

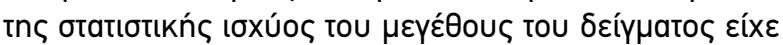

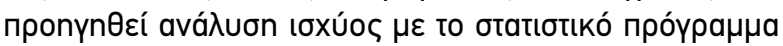

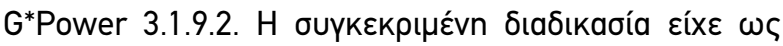

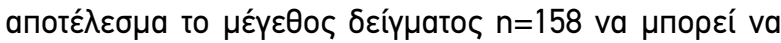

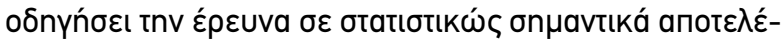

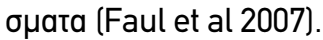

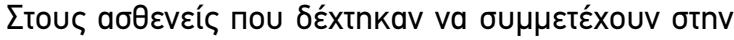

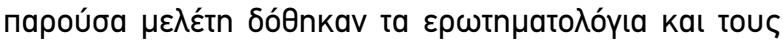

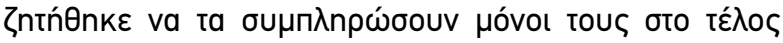

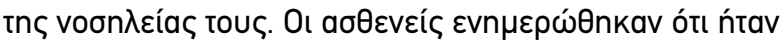

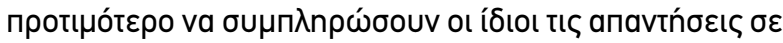

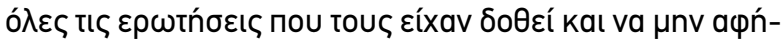

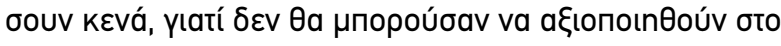

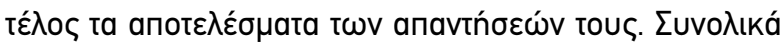

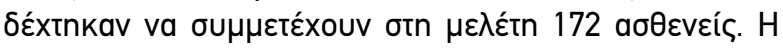

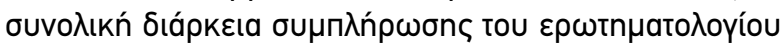

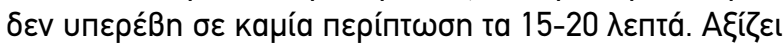

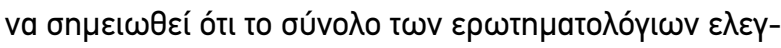

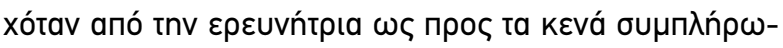

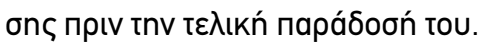

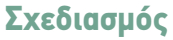

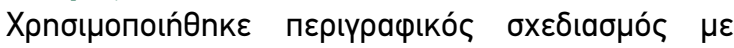

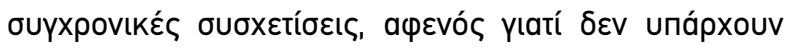

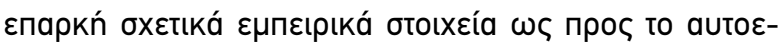

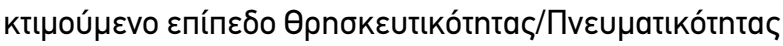

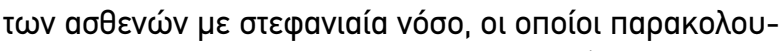

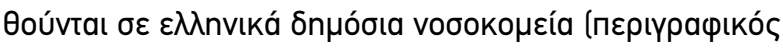

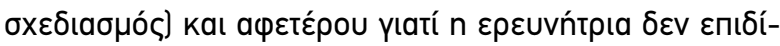

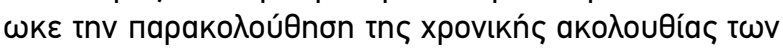

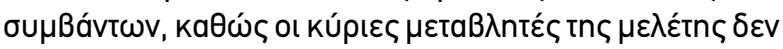

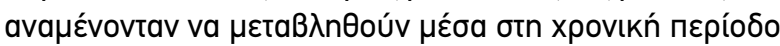

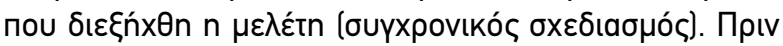

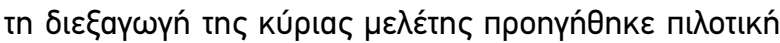

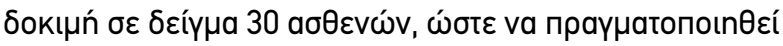

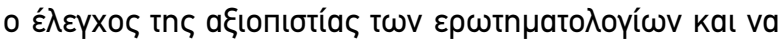

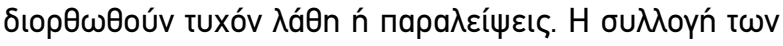

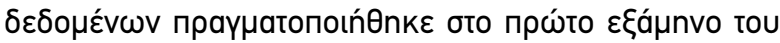
2018.

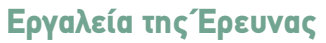

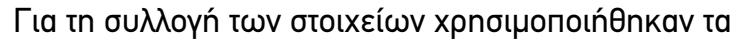

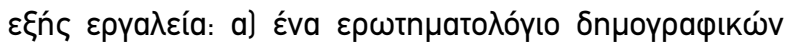

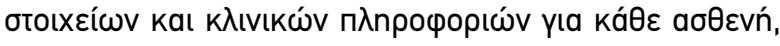

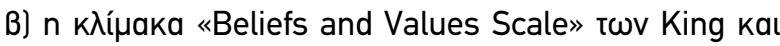

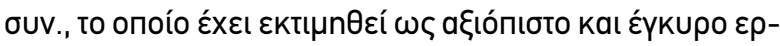

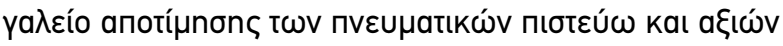

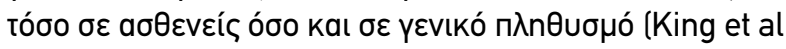

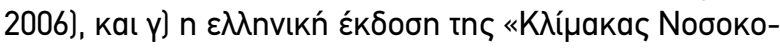

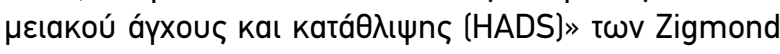

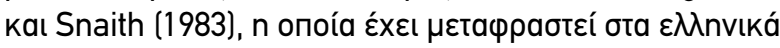
anó touৎ Мıxónou

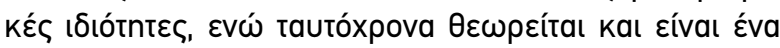

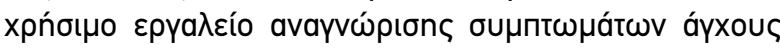

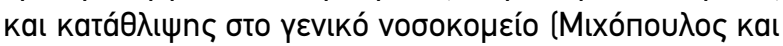
ouv 2007).

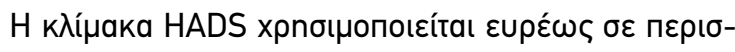

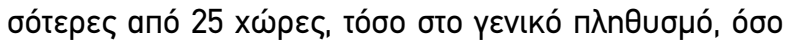

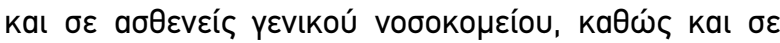

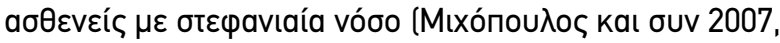

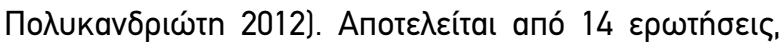

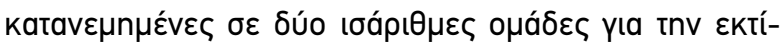

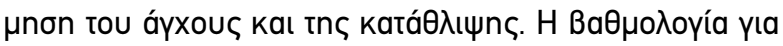

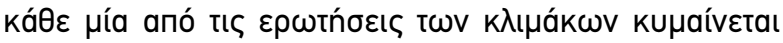

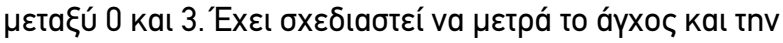

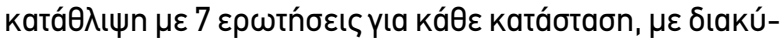

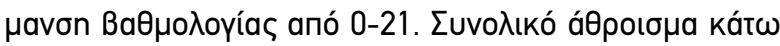

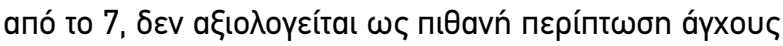

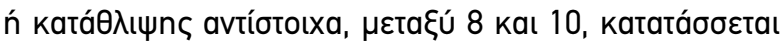

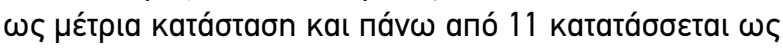

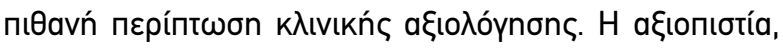

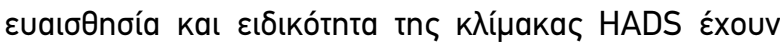

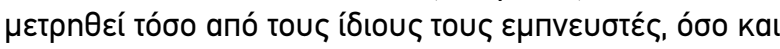

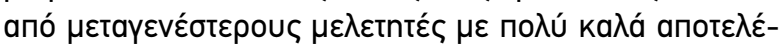
бнata.

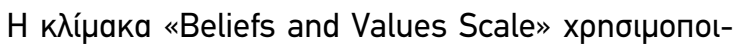

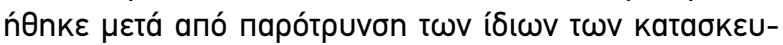

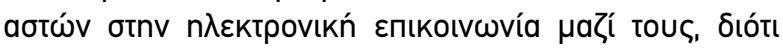

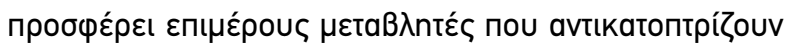




\section{EPEYNHTIKA APOPA}

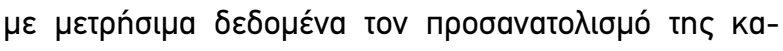

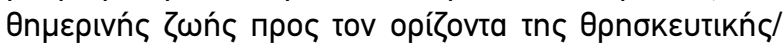

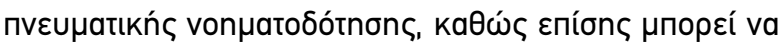

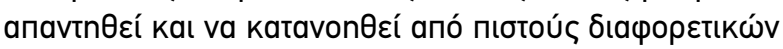

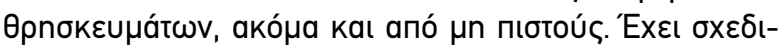

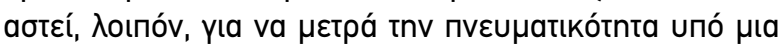

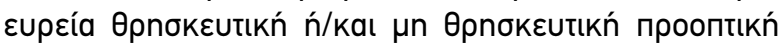

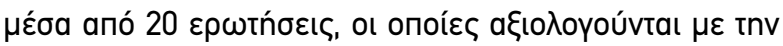

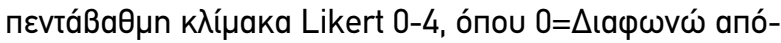

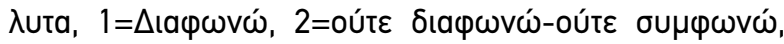

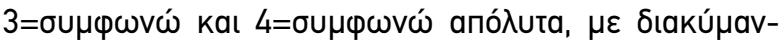

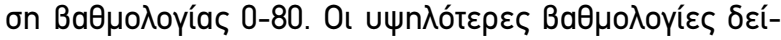

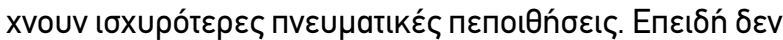

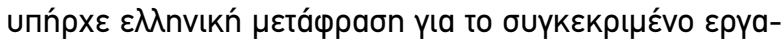

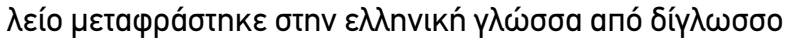

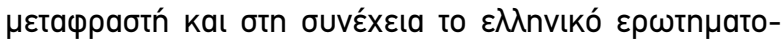

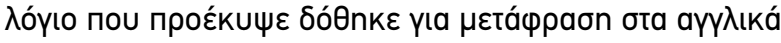

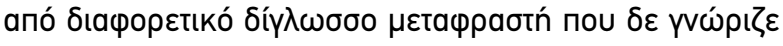

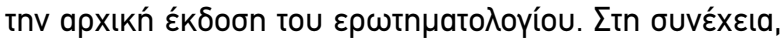

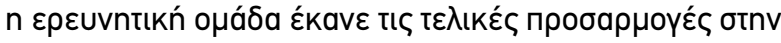

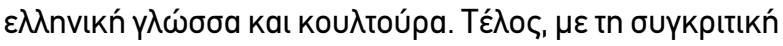

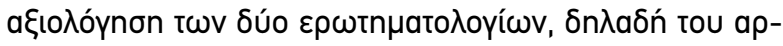

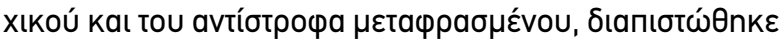

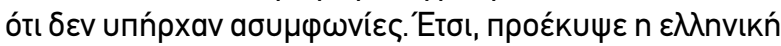

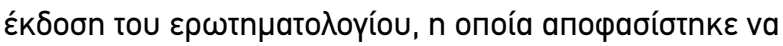

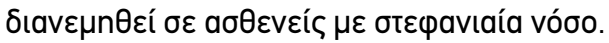

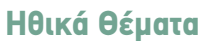

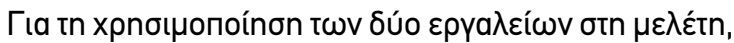

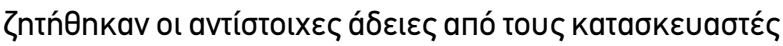

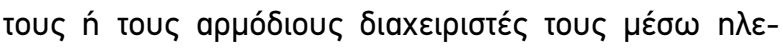

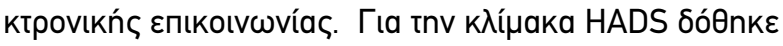

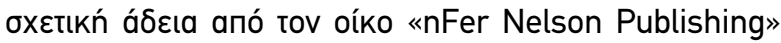

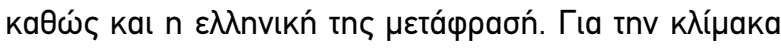

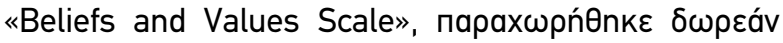

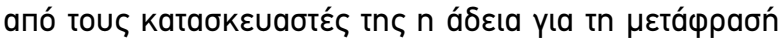

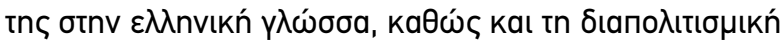

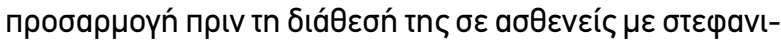

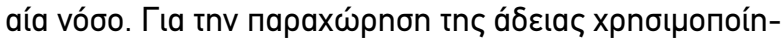

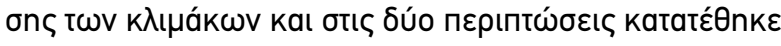

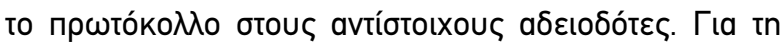

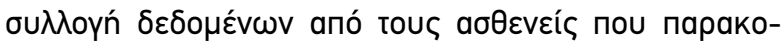

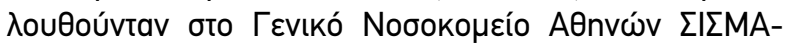

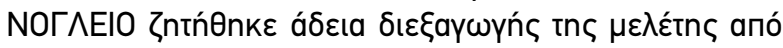

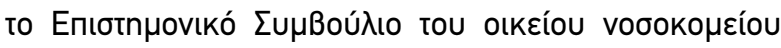

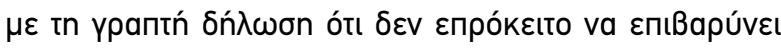

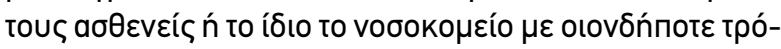

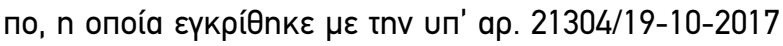

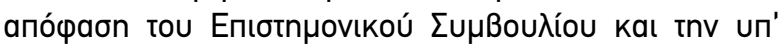

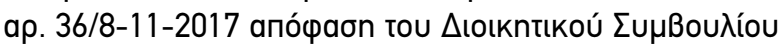

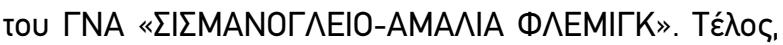

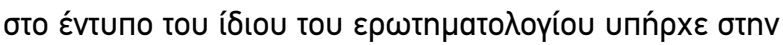

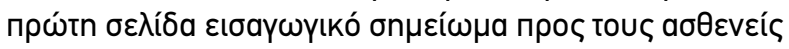

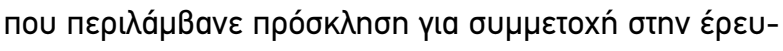

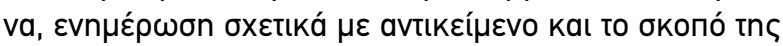

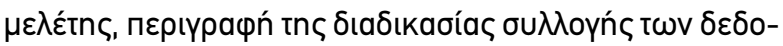

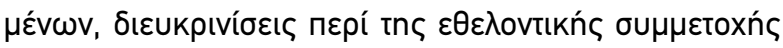

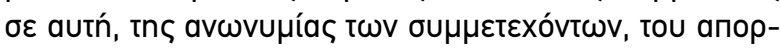

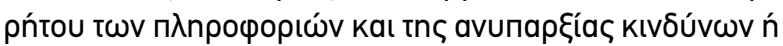

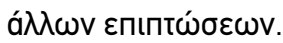

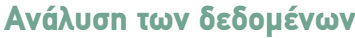

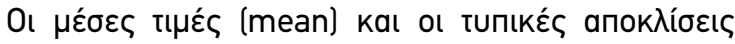

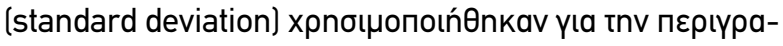

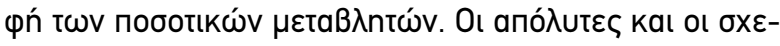

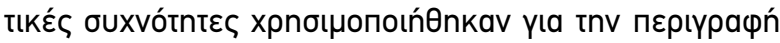

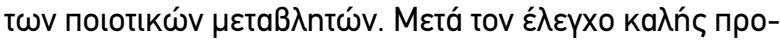

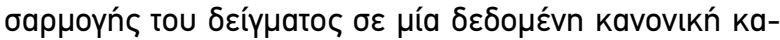

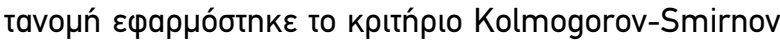

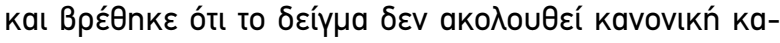

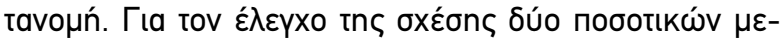

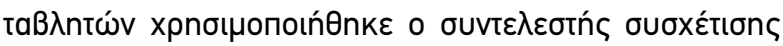

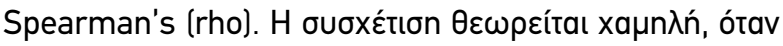

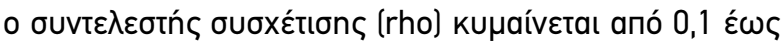

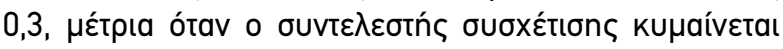

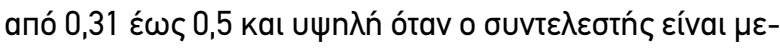

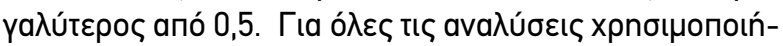

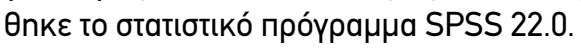

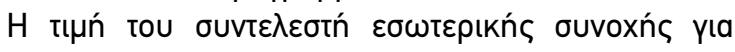

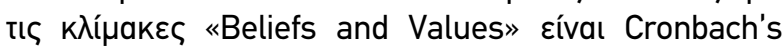
alpha $=0,928$, yıa tnv HADS-Anxiety eívaı Cronbach's alpha $=0,838$ ka үıа tnv k $\lambda i ́ \mu a k a$ HADS-Depression $\varepsilon i ́-$

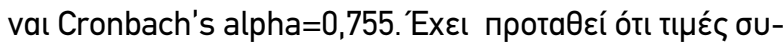

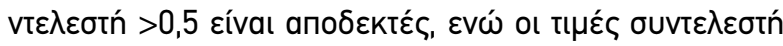

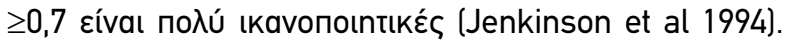

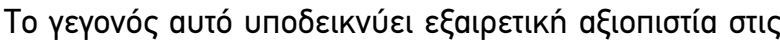

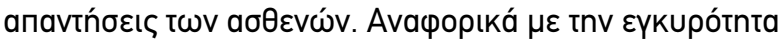

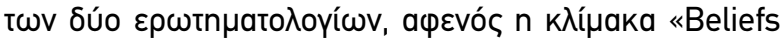

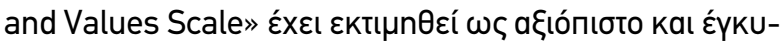

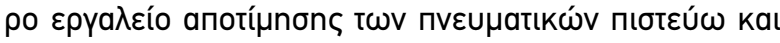

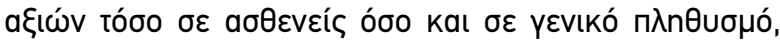

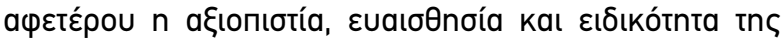

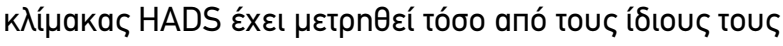

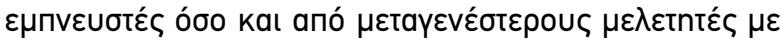

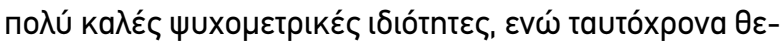

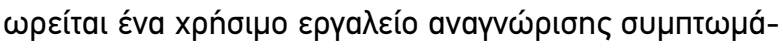

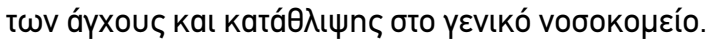

\section{АПОТЕАЕЕMATA}

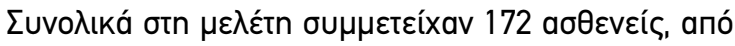
tous onoíous ol 149 (86,6\%) ńtav ávopes, ol 113 (64,9\%)

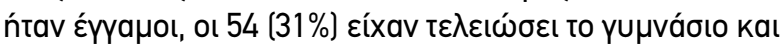
oเ $59(33,9 \%)$ ńtav $\varepsilon \lambda \varepsilon u ́ \theta \varepsilon \rho o l ~ \varepsilon п a \gamma \gamma \varepsilon \lambda \mu a t i ́ \varepsilon \varsigma$. To $\mu \varepsilon \gamma a \lambda u ́ t \varepsilon-$

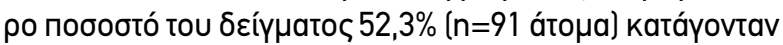

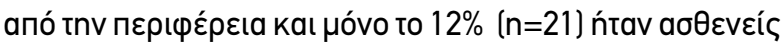

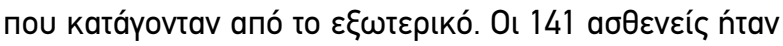




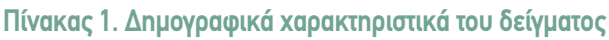

\begin{tabular}{|c|c|}
\hline 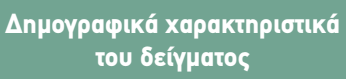 & $\mathbf{N}(\%)$ \\
\hline \multicolumn{2}{|l|}{ Фúxo } \\
\hline 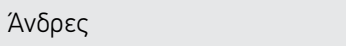 & $149(85,6)$ \\
\hline Гuvaíkદৎ & $23(13,2)$ \\
\hline 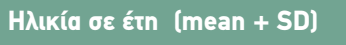 & $62,85+12$ \\
\hline [min-max] & $(32-91)$ \\
\hline 32-52 દ́đก & $39(22,4)$ \\
\hline 53-67 દ́đn & $69(39,7)$ \\
\hline 68-78źtn & $49(28,2)$ \\
\hline <79 દ́tn & $15(8,6)$ \\
\hline \multicolumn{2}{|l|}{ Otkoyevelakń katáotaơn } \\
\hline Аүанос & $11(6,3)$ \\
\hline 'Еүуанос & $113(64,9)$ \\
\hline 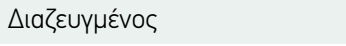 & $16(9,2)$ \\
\hline Xńpos & $18(10,3)$ \\
\hline 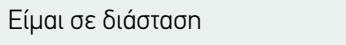 & $1(0,6)$ \\
\hline 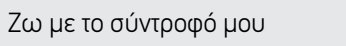 & $13(7,5)$ \\
\hline \multicolumn{2}{|l|}{ Tónos Kataywyńs } \\
\hline Aтtıkń & $58(33,3)$ \\
\hline Пદрıфદ́рદıа & $91(52,3)$ \\
\hline 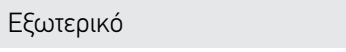 & $21(12,1)$ \\
\hline 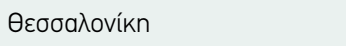 & $1(0,6)$ \\
\hline
\end{tabular}

\begin{tabular}{|c|c|}
\hline 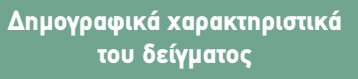 & $\mathbf{N}[\%]$ \\
\hline \multicolumn{2}{|l|}{ 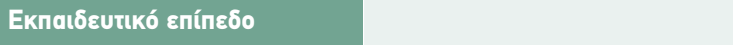 } \\
\hline Аүра́ннатос & $1(0,6)$ \\
\hline$\Delta$ nнотাкó & $35(20,1)$ \\
\hline Гuнváoı & $54(31)$ \\
\hline ^úKદાO & $24(13,8)$ \\
\hline IEK & $4(2,3)$ \\
\hline TEI & $22(12,6)$ \\
\hline 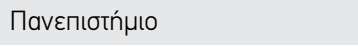 & $28(16,1)$ \\
\hline Metantuxıakó & $3(1,7)$ \\
\hline АІठакторıкó & $1(0,6)$ \\
\hline \multicolumn{2}{|l|}{ 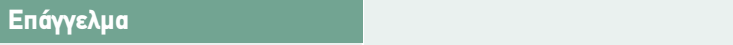 } \\
\hline 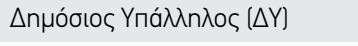 & $16(9,2)$ \\
\hline 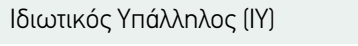 & $44(25,3)$ \\
\hline ¿uvtakıoúxoৎ & $47(27)$ \\
\hline 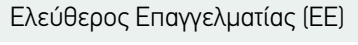 & $59(33,9)$ \\
\hline Oıкıакá & $6(3,4)$ \\
\hline \multicolumn{2}{|l|}{ 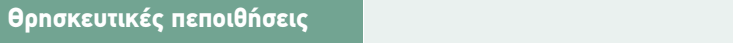 } \\
\hline АӨદ૦ৎ & $18(10,3)$ \\
\hline 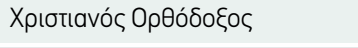 & $141(81)$ \\
\hline Xpıotıavós KaӨo入ıкóৎ & $2(1,1)$ \\
\hline 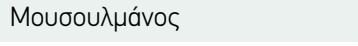 & $2(1,1)$ \\
\hline AMlo & $2(6,7)$ \\
\hline
\end{tabular}

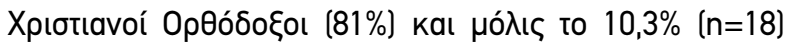

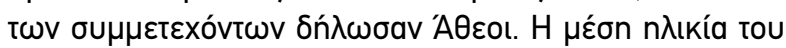

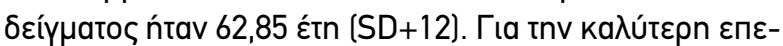

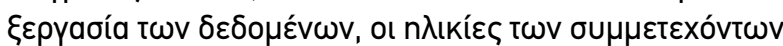

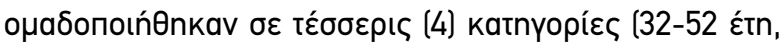

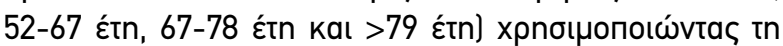

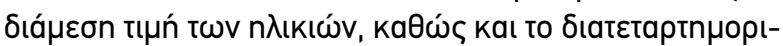

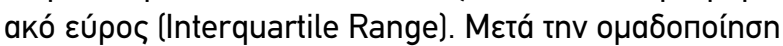

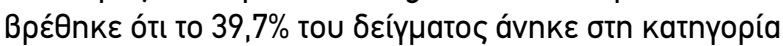

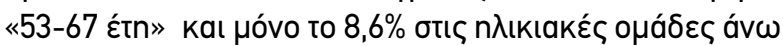

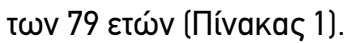

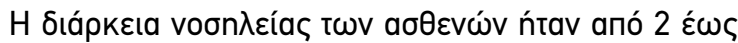

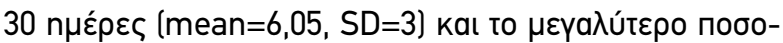

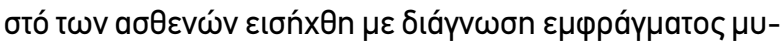
okapó́ou $\mu \varepsilon$ aváonaon tou ST (STEMI) $(46,6 \%, \mathrm{n}=81)$. To

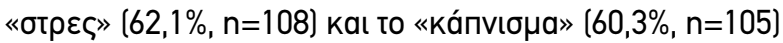

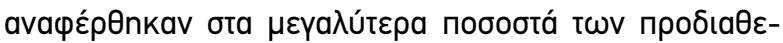

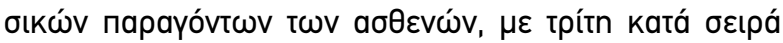

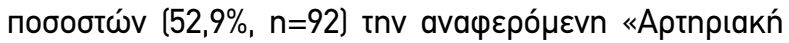

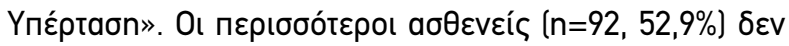

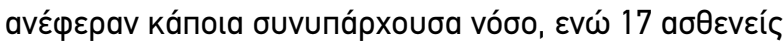

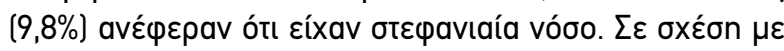

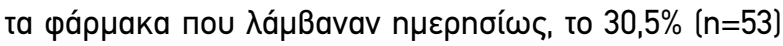

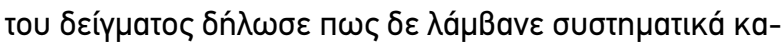

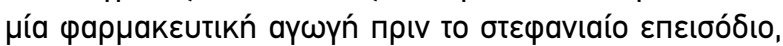

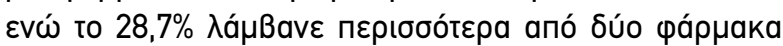

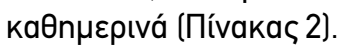

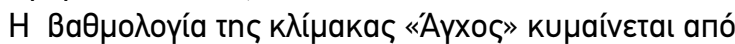

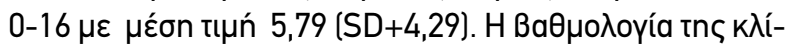

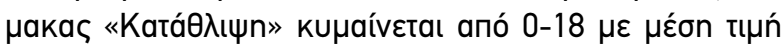

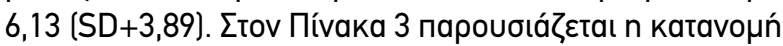

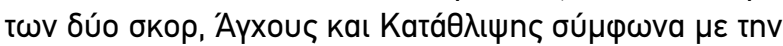

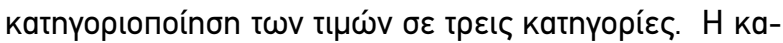

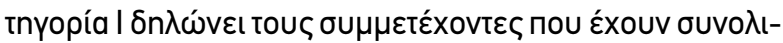

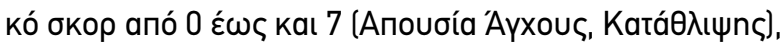

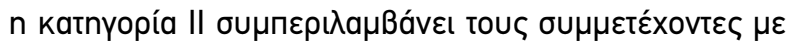

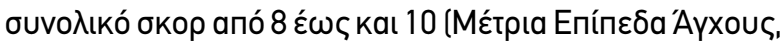

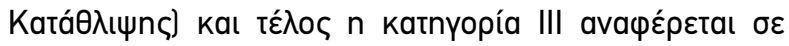




\section{EPEYNHTIKA APOPA}

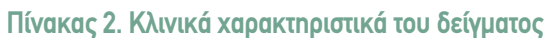

\begin{tabular}{|c|c|}
\hline 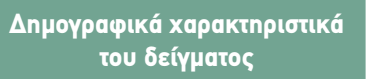 & $\mathbf{N}[\%]$ \\
\hline 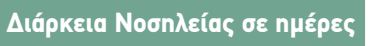 & $6,05+3$ \\
\hline (mean + SD) (min-max) & $(2-30)$ \\
\hline \multicolumn{2}{|l|}{ Пароúбa vóoos } \\
\hline 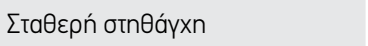 & $13(7,5)$ \\
\hline AбтаӨń бтnӨáyxn & $17(9,8)$ \\
\hline STEMI & $81(46,6)$ \\
\hline non STEMI & $51(29,3)$ \\
\hline \multicolumn{2}{|l|}{ ¿uvunápxouoa vódos } \\
\hline Kauía & $92(52,9)$ \\
\hline 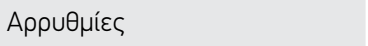 & $1(0,6)$ \\
\hline Ва入ßıઠопа́Өદıа & $4(2,3)$ \\
\hline 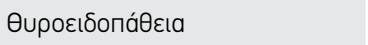 & $11(6,3)$ \\
\hline 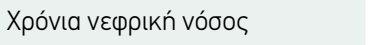 & $10(5,7)$ \\
\hline 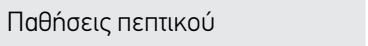 & $4(2,3)$ \\
\hline 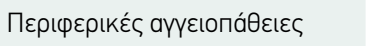 & $3(1,7)$ \\
\hline 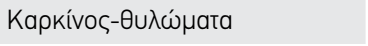 & $7(4)$ \\
\hline 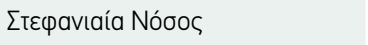 & $17(9,8)$ \\
\hline ХАП & $6(3,4)$ \\
\hline \multicolumn{2}{|l|}{ ПробıаӨвoukoí парáүovtes } \\
\hline 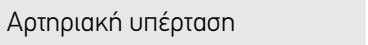 & $92(52,9)$ \\
\hline 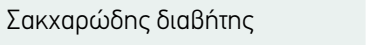 & $46(26,4)$ \\
\hline$\Delta \cup \sigma \lambda ı п เ \delta a ı \mu i ́ a$ & $87(50)$ \\
\hline Stress & $108(62,1)$ \\
\hline 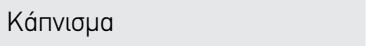 & $105(60,3)$ \\
\hline Пахиоаркі́a & $26(14,9)$ \\
\hline \multicolumn{2}{|l|}{ Фарнаквutukń аүळүи́ } \\
\hline Kapía & $53(30,5)$ \\
\hline 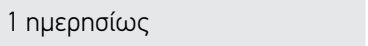 & $36(20,7)$ \\
\hline 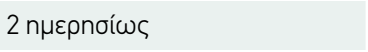 & $30(17,2)$ \\
\hline 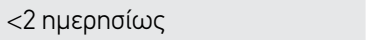 & $50(28,7)$ \\
\hline
\end{tabular}

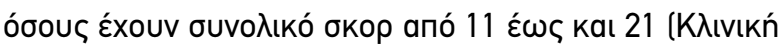

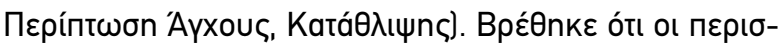

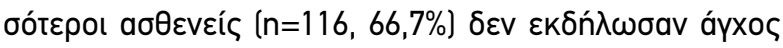

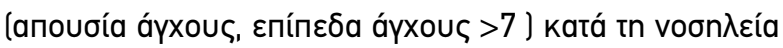

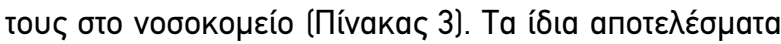

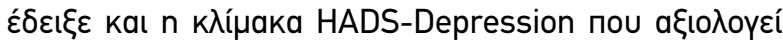

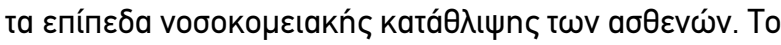

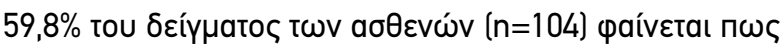

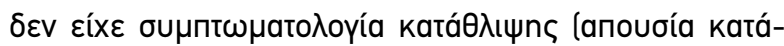

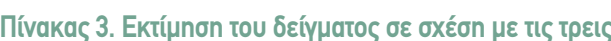

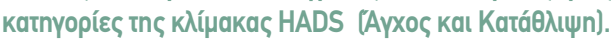

\begin{tabular}{|c|c|}
\hline $\begin{array}{c}\text { HADS -Anxiety Ayxos } \\
\text { Typés: 0-16 } \\
\text { mean= 5,79 }(S D+4,29)\end{array}$ & \multirow[t]{2}{*}{$\mathbf{N}(\%)$} \\
\hline Katnyopís & \\
\hline I & $116(66,7)$ \\
\hline II & $29(16,7)$ \\
\hline III & $27(15,5)$ \\
\hline 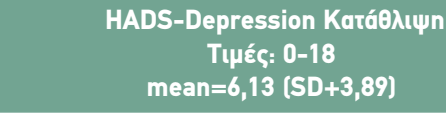 & \multirow[t]{2}{*}{$\mathbf{N}(\%)$} \\
\hline Katnyopies & \\
\hline I & $104(59,8)$ \\
\hline II & 47 (27) \\
\hline III & $21(12,1)$ \\
\hline
\end{tabular}

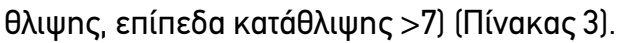

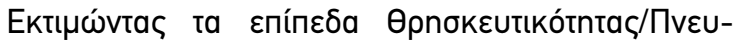

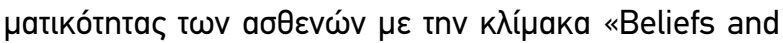

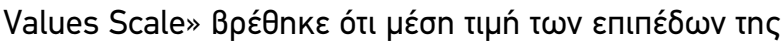

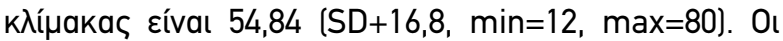

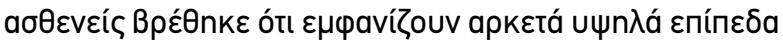

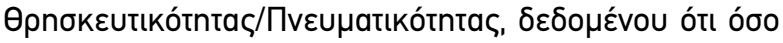

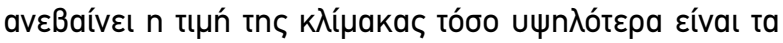

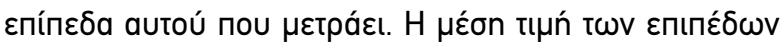

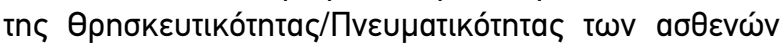

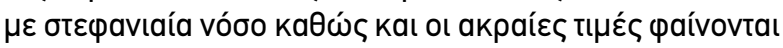
бто Exń $\mu$ a 1.

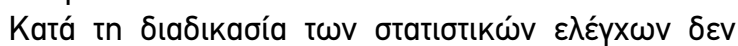

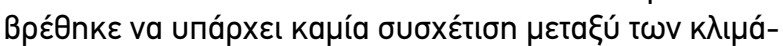

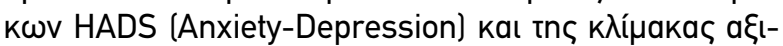

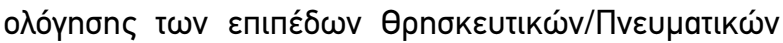

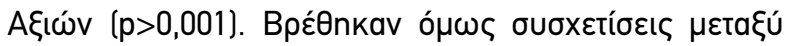

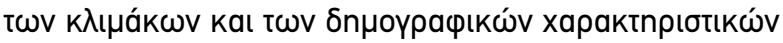

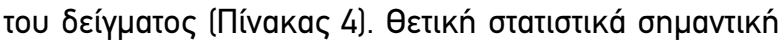

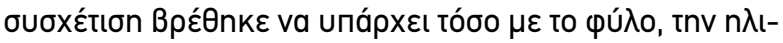

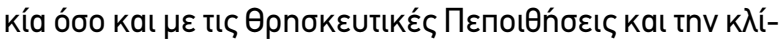

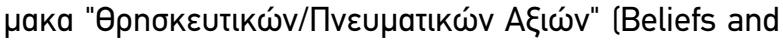

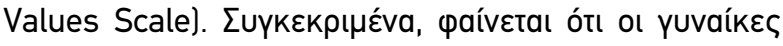

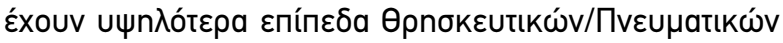

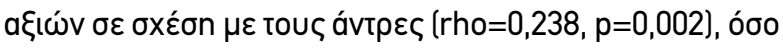

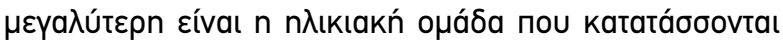

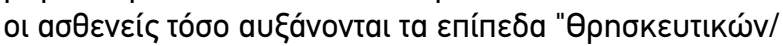

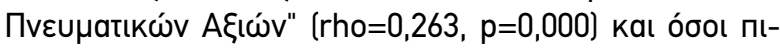

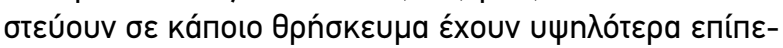

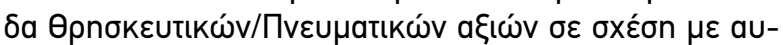

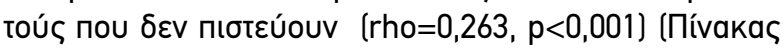
4).

Katá tn ouoxźtion tou Ayxous (HADS Anxiety) $\mu \varepsilon$ 


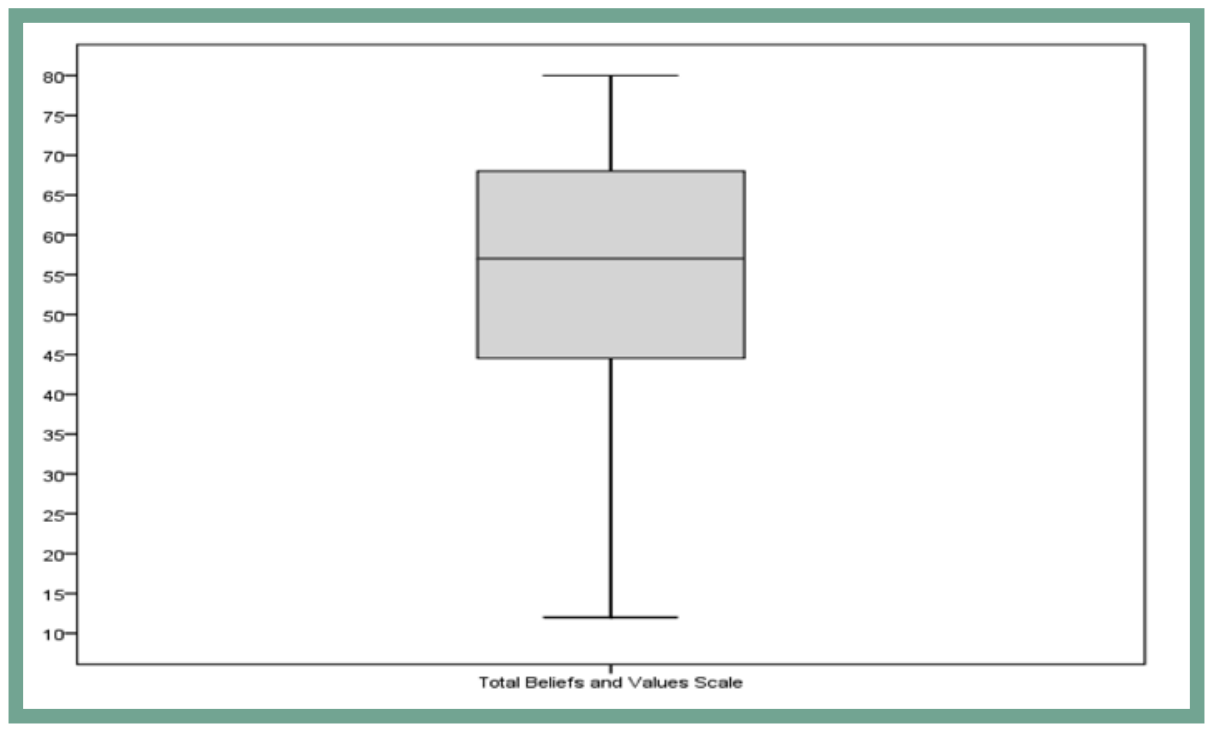

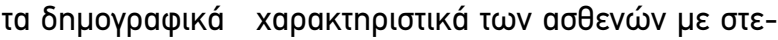

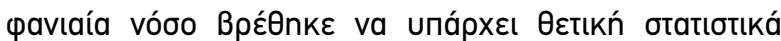

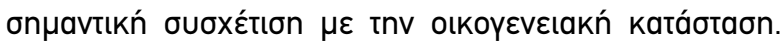

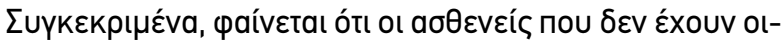

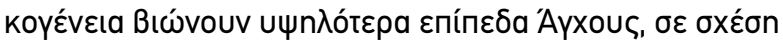

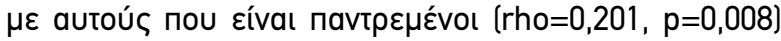

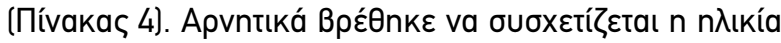

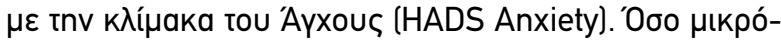

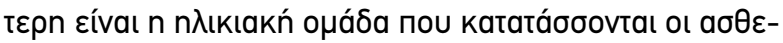

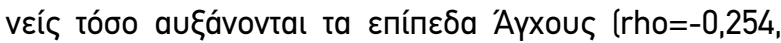
$\mathrm{p}=0,001$ ) (Пívakas 4).

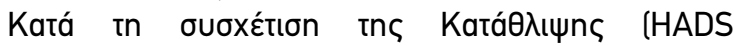

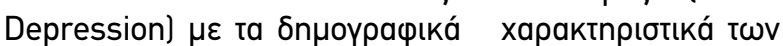

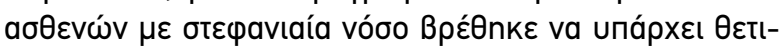

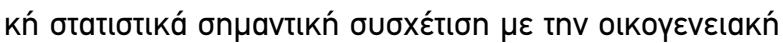

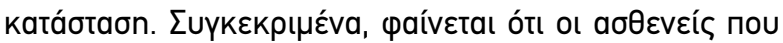

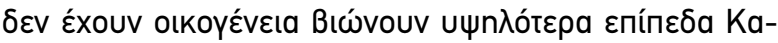

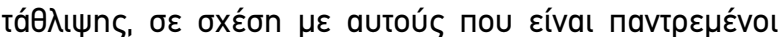

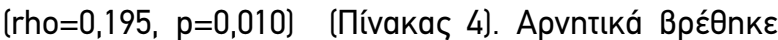

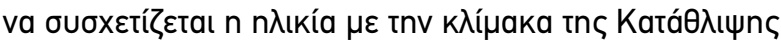

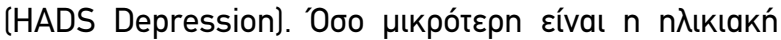
o $\varepsilon \Pi i ́ n \varepsilon \delta a$ Katá $\theta \lambda ı$ ıs (rho=-0,222, p=0,003) (Пívakas 4).

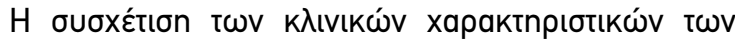

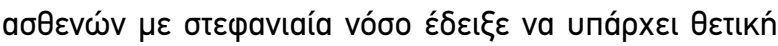

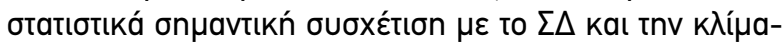

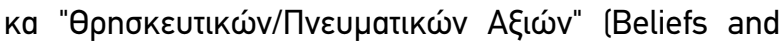

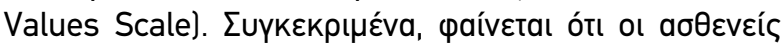

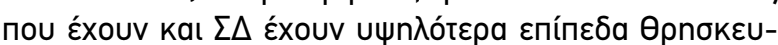

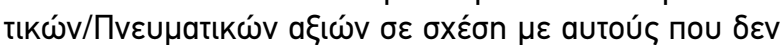

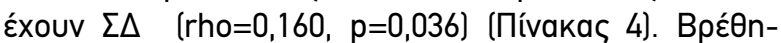

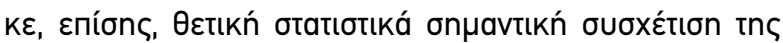

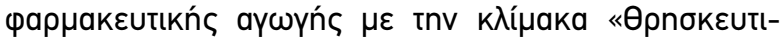

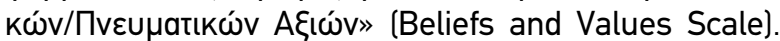

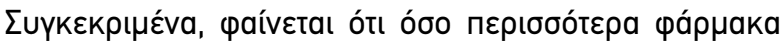

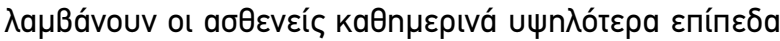

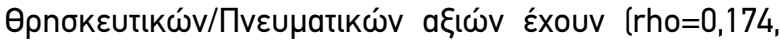
$\mathrm{p}=0,023)$ (Пі́vakas 4).

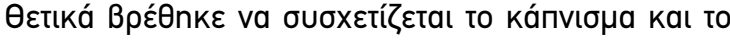

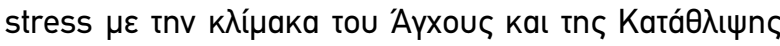
(HADS Anxiety-Depression). 'Oool kanvíZouv paívetaı va

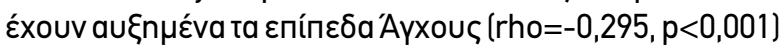

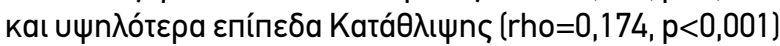

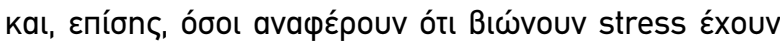

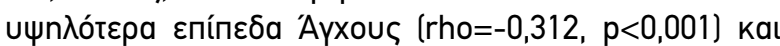

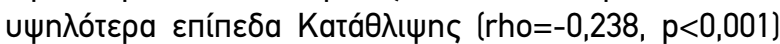

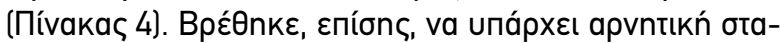

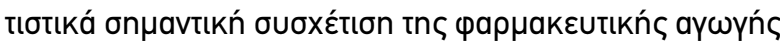

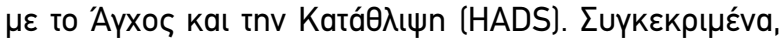

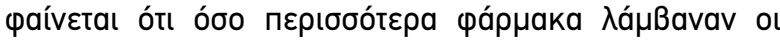

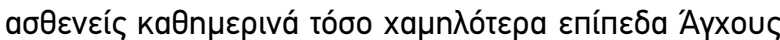

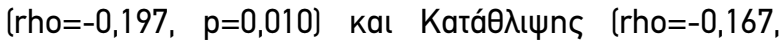

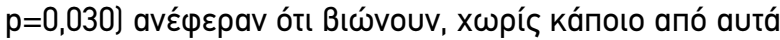
va zívaı avtıkata $\theta \lambda$ ıпtıкó (Пívakas 4).

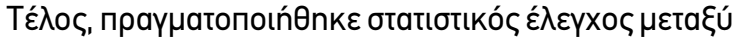

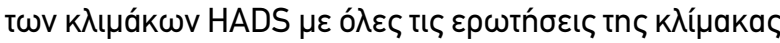

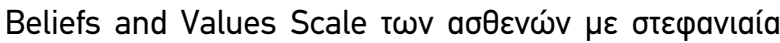

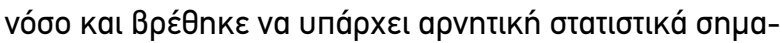

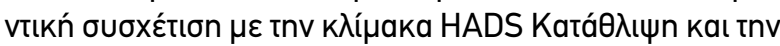

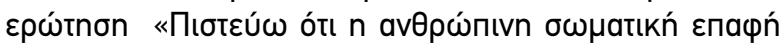

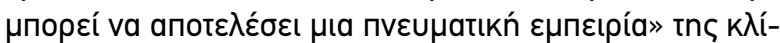

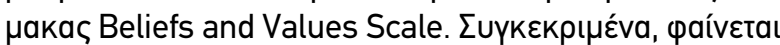

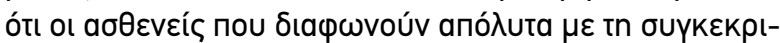

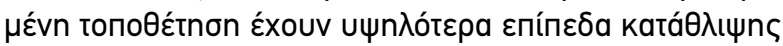




\section{EPEYNHTIKA APOPA}

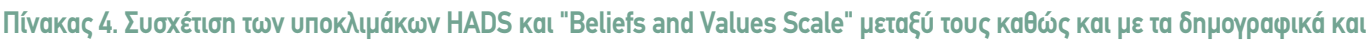

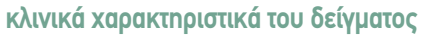

\begin{tabular}{|c|c|c|c|c|}
\hline 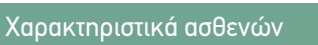 & Spearman's rho & Total Beliefs Values Scale & HADS Anxiety ('Ayxoৎ) & 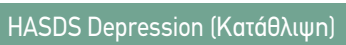 \\
\hline \multirow[t]{3}{*}{ Total Beliefs Values Scale } & $\begin{array}{l}\text { rho } \\
\text { Correlation Coefficient }\end{array}$ & 1,000 & $-0,076$ & $-0,124$ \\
\hline & Sig. (2-tailed) & . & 0,320 & 0,105 \\
\hline & $\mathrm{N}$ & 172 & 172 & 172 \\
\hline \multirow[t]{3}{*}{$\begin{array}{l}\text { HADS Anxiety } \\
\text { (Ayxo૬) }\end{array}$} & $\begin{array}{l}\text { rho } \\
\text { Correlation Coefficient }\end{array}$ & $-0,076$ & 1,00 & $0,707^{* *}$ \\
\hline & Sig. (2-tailed) & 0,320 & . & 0,001 \\
\hline & $\mathrm{N}$ & 172 & 172 & 172 \\
\hline \multirow[t]{3}{*}{$\begin{array}{l}\text { HADS Depression } \\
\text { (Katá } \lambda_{(\Psi n)}\end{array}$} & $\begin{array}{l}\text { rho } \\
\text { Correlation Coefficient }\end{array}$ & $-0,124$ & $0,707^{* *}$ & 1,00 \\
\hline & Sig. (2-tailed) & 0,105 & 0,000 & . \\
\hline & $\mathrm{N}$ & 172 & 172 & 172 \\
\hline \multirow[t]{3}{*}{ Фúlo } & $\begin{array}{l}\text { rho } \\
\text { Correlation Coefficient }\end{array}$ & $0,238^{* *}$ & $-0,108$ & $-0,108$ \\
\hline & Sig. (2-tailed) & 0,002 & 0,160 & 0,157 \\
\hline & $\mathrm{N}$ & 172 & 172 & 172 \\
\hline \multirow[t]{3}{*}{ 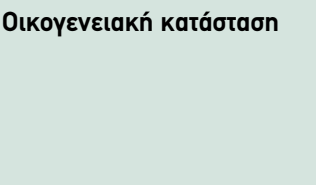 } & $\begin{array}{l}\text { rho } \\
\text { Correlation Coefficient }\end{array}$ & $-0,146$ & $0.201^{* *}$ & $0,195^{* *}$ \\
\hline & Sig. (2-tailed) & 0,056 & 0,008 & 0,010 \\
\hline & N & 172 & 172 & 172 \\
\hline \multirow[t]{3}{*}{ 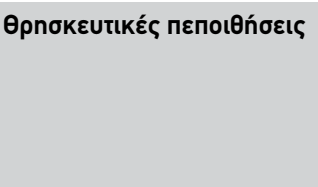 } & $\begin{array}{l}\text { rho } \\
\text { Correlation Coefficient }\end{array}$ & $0,263^{* *}$ & $-0,032$ & \\
\hline & Sig. (2-tailed) & 0,000 & 0.679 & \\
\hline & $N$ & 172 & 172 & \\
\hline \multirow[t]{3}{*}{ 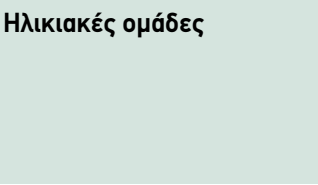 } & $\begin{array}{l}\text { rho } \\
\text { Correlation Coefficient }\end{array}$ & $0,181^{*}$ & $-0,254^{* *}$ & $-0,026$ \\
\hline & Sig. (2-tailed) & 0,017 & 0,001 & 0,736 \\
\hline & $\mathrm{N}$ & 172 & 172 & 172 \\
\hline \multirow[t]{3}{*}{ 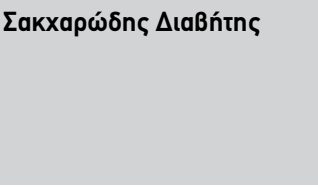 } & $\begin{array}{l}\text { rho } \\
\text { Correlation Coefficient }\end{array}$ & $0,160^{*}$ & $-0,057$ & \\
\hline & Sig. (2-tailed) & 0,036 & 0,455 & \\
\hline & $\mathrm{N}$ & 172 & 172 & \\
\hline \multirow[t]{3}{*}{ Kánvioua } & $\begin{array}{l}\text { rho } \\
\text { Correlation Coefficient }\end{array}$ & 0,013 & $0.295^{* *}$ & $0,174^{* *}$ \\
\hline & Sig. (2-tailed) & 0,867 & 0,000 & 0,023 \\
\hline & $\mathrm{N}$ & 172 & 172 & 172 \\
\hline \multirow[t]{3}{*}{ Stress } & $\begin{array}{l}\text { rho } \\
\text { Correlation Coefficient }\end{array}$ & 0,076 & $0,312^{* *}$ & $0,283^{* *}$ \\
\hline & Sig. (2-tailed) & 0,319 & 0,000 & 0,000 \\
\hline & $\mathrm{N}$ & 172 & 172 & 172 \\
\hline \multirow[t]{3}{*}{ 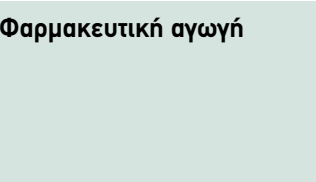 } & $\begin{array}{l}\text { rho } \\
\text { Correlation Coefficient }\end{array}$ & $0,174^{*}$ & $-0,197^{* *}$ & $-0,167^{* *}$ \\
\hline & Sig. (2-tailed) & 0,023 & 0,010 & 0,030 \\
\hline & $\mathrm{N}$ & 169 & 169 & 169 \\
\hline
\end{tabular}

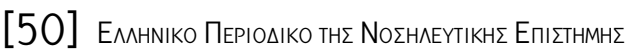




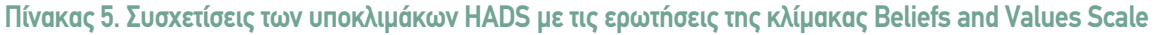

\begin{tabular}{|c|c|c|c|}
\hline Beliefs and Values Scale & Spearman's rho & HADS AYxoৎ & HADS KatáӨ $\lambda \iota \psi n$ \\
\hline \multirow[t]{3}{*}{ 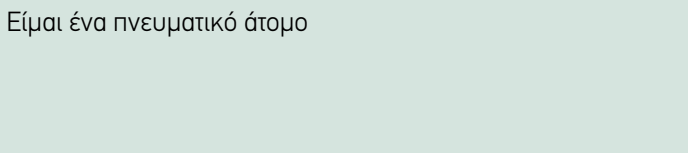 } & Correlation Coefficient & $-0,076$ & $-0,107$ \\
\hline & Sig. (2-tailed) & 0,322 & 0,162 \\
\hline & N & 172 & 172 \\
\hline \multirow{3}{*}{ 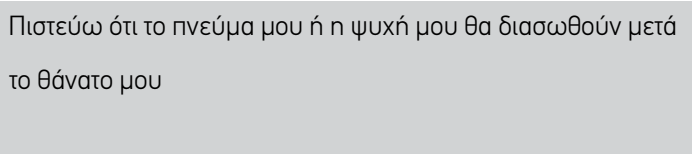 } & Correlation Coefficient & 0,034 & $-0,019$ \\
\hline & Sig. (2-tailed) & 0,661 & 0,802 \\
\hline & $\mathrm{N}$ & 172 & 172 \\
\hline \multirow[t]{3}{*}{ 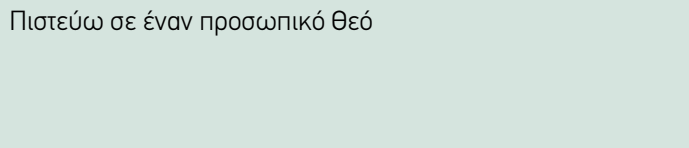 } & Correlation Coefficient & 0,063 & $-0,034$ \\
\hline & Sig. (2-tailed) & 0,410 & 0,660 \\
\hline & N & 172 & 172 \\
\hline \multirow[t]{3}{*}{ 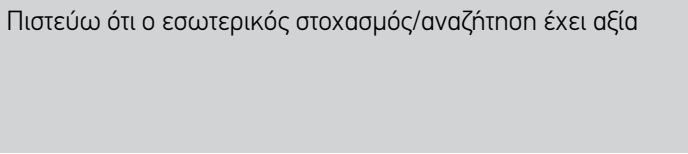 } & Correlation Coefficient & 0,048 & $-0,038$ \\
\hline & Sig. (2-tailed) & 0,533 & 0,618 \\
\hline & $\mathrm{N}$ & 172 & 172 \\
\hline \multirow[t]{3}{*}{ 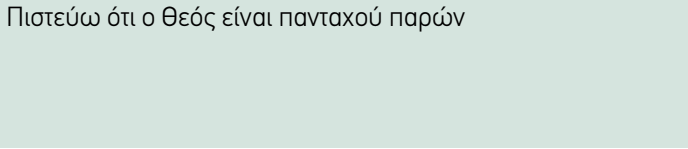 } & Correlation Coefficient & 0,113 & 0,015 \\
\hline & Sig. (2-tailed) & 0,140 & 0,842 \\
\hline & N & 172 & 172 \\
\hline \multirow{3}{*}{ 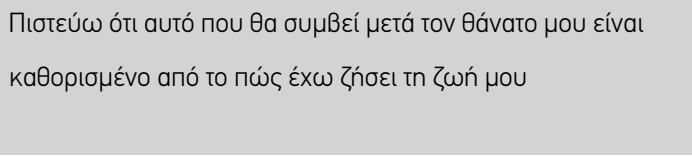 } & Correlation Coefficient & 0,019 & $-0,122$ \\
\hline & Sig. (2-tailed) & 0,808 & 0,109 \\
\hline & N & 172 & 172 \\
\hline \multirow[t]{3}{*}{ 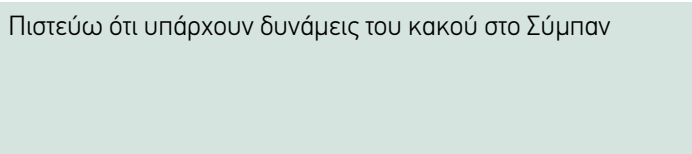 } & Correlation Coefficient & 0,011 & 0,080 \\
\hline & Sig. (2-tailed) & 0,889 & 0,295 \\
\hline & $\mathrm{N}$ & 172 & 172 \\
\hline \multirow{3}{*}{ 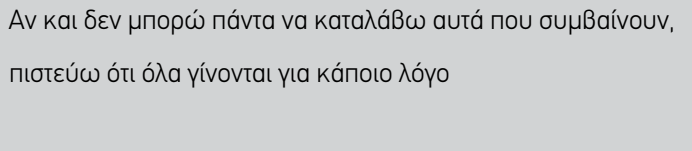 } & Correlation Coefficient & 0,129 & 0,053 \\
\hline & Sig. (2-tailed) & 0,091 & 0,491 \\
\hline & $\mathrm{N}$ & 172 & 172 \\
\hline \multirow{3}{*}{ 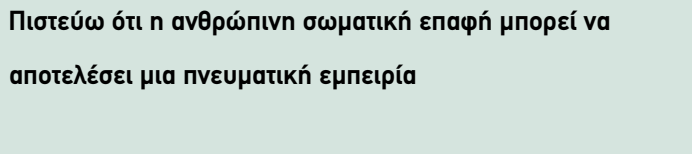 } & Correlation Coefficient & $-0,129$ & $-0,195^{*}$ \\
\hline & Sig. (2-tailed) & 0,091 & 0,010 \\
\hline & $\mathrm{N}$ & 172 & 172 \\
\hline \multirow{3}{*}{ 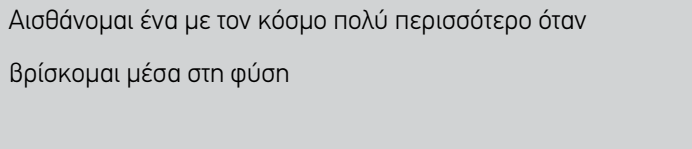 } & Correlation Coefficient & $-0,098$ & $-0,139$ \\
\hline & Sig. (2-tailed) & 0,202 & 0,069 \\
\hline & $\mathrm{N}$ & 172 & 172 \\
\hline \multirow[t]{3}{*}{ 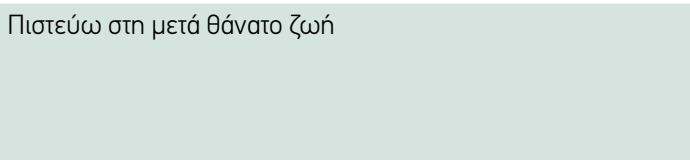 } & Correlation Coefficient & 0,009 & $-0,120$ \\
\hline & Sig. (2-tailed) & 0,907 & 0,118 \\
\hline & $\mathrm{N}$ & 172 & 172 \\
\hline \multirow[t]{3}{*}{ 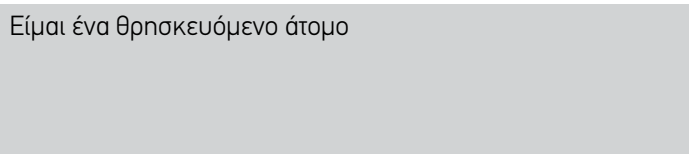 } & Correlation Coefficient & 0,091 & 0,081 \\
\hline & Sig. (2-tailed) & 0,237 & 0,294 \\
\hline & $\mathrm{N}$ & 172 & 172 \\
\hline \multirow[t]{3}{*}{ 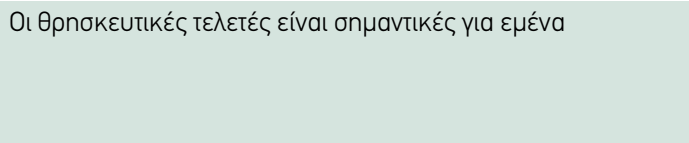 } & Correlation Coefficient & $-0,010$ & $-0,025$ \\
\hline & Sig. (2-tailed) & 0,900 & 0,747 \\
\hline & $\mathrm{N}$ & 172 & 172 \\
\hline
\end{tabular}




\section{EPEYNHTIKA APOPA}

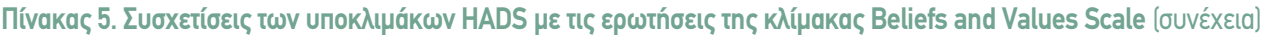

\begin{tabular}{|c|c|c|c|}
\hline Beliefs and Values Scale & Spearman's rho & HADS Ayxoৎ & 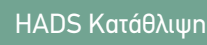 \\
\hline \multirow[t]{3}{*}{ 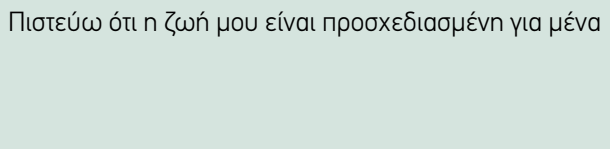 } & Correlation Coefficient & 0,039 & 0,053 \\
\hline & Sig. (2-tailed) & 0,613 & 0,489 \\
\hline & $\mathrm{N}$ & 172 & 172 \\
\hline \multirow[t]{3}{*}{ 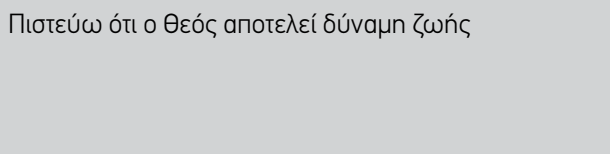 } & Correlation Coefficient & 0,073 & $-0,027$ \\
\hline & Sig. (2-tailed) & 0,342 & 0,729 \\
\hline & $\mathrm{N}$ & 172 & 172 \\
\hline \multirow{3}{*}{ 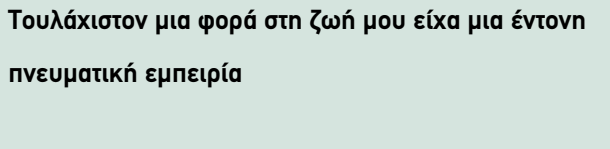 } & Correlation Coefficient & $-0,219^{* *}$ & $-0,184^{*}$ \\
\hline & Sig. (2-tailed) & 0,004 & 0,016 \\
\hline & $\mathrm{N}$ & 172 & 172 \\
\hline \multirow[t]{3}{*}{ 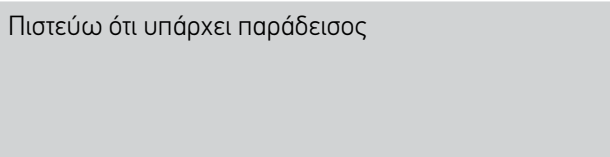 } & Correlation Coefficient & 0,065 & $-0,026$ \\
\hline & Sig. (2-tailed) & 0,396 & 0,732 \\
\hline & N & 172 & 172 \\
\hline \multirow[t]{3}{*}{ 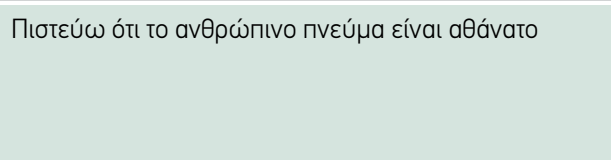 } & Correlation Coefficient & $-0,008$ & $-0,100$ \\
\hline & Sig. (2-tailed) & 0,912 & 0,194 \\
\hline & $\mathrm{N}$ & 172 & 172 \\
\hline \multirow[t]{3}{*}{ 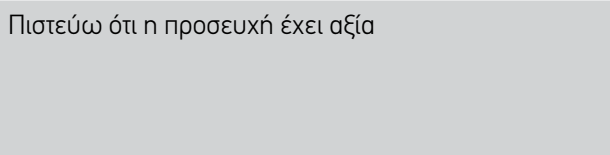 } & Correlation Coefficient & .042 & $-0,001$ \\
\hline & Sig. (2-tailed) & 0,581 & 0,986 \\
\hline & $\mathrm{N}$ & 172 & 172 \\
\hline \multirow[t]{3}{*}{ 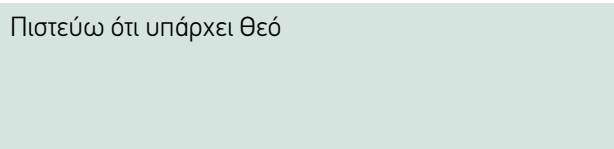 } & Correlation Coefficient & 0,080 & $-0,013$ \\
\hline & Sig. (2-tailed) & 0,296 & 0,868 \\
\hline & $\mathrm{N}$ & 172 & 172 \\
\hline
\end{tabular}

**. Correlation is significant at the 0,01 level (2-tailed).

*. Correlation is significant at the 0,05 level (2-tailed).

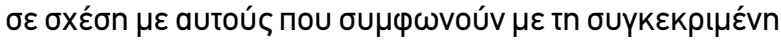
toпо日étnon (rho=-0,195, p=0,010) (Пívakas 5). Eníons,

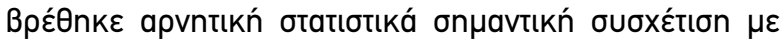

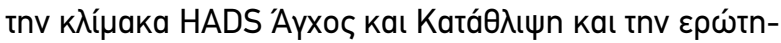

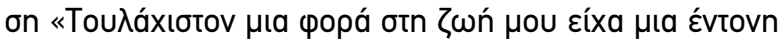

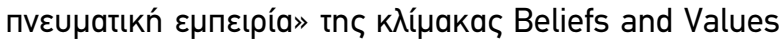

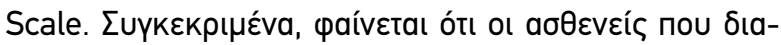

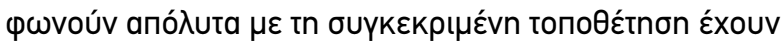

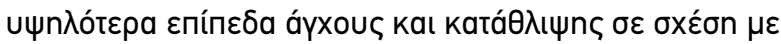

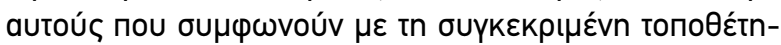
on ('Aүxoৎ rho=-0,219, $p=0,004$, Katá $\theta \lambda ı \Psi n$ rho=-0,184, $\mathrm{p}=0,016)$ (Пívakas 5).

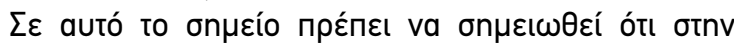

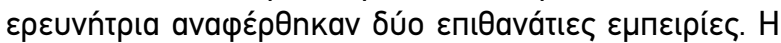

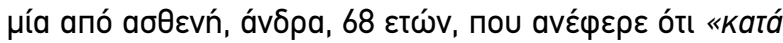

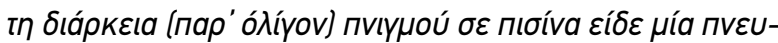

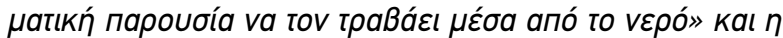

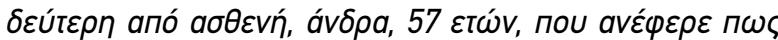

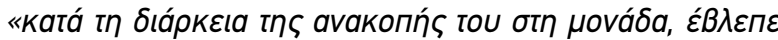

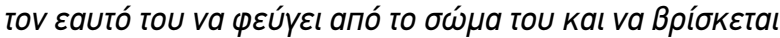

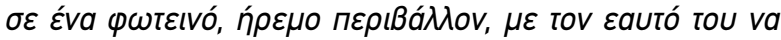
$v i \omega ́ \theta \varepsilon l$ yaגńvn».

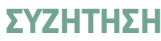

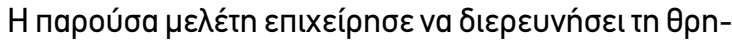

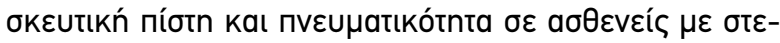

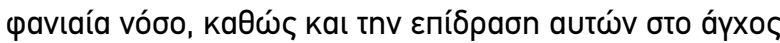

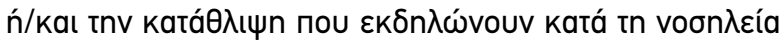

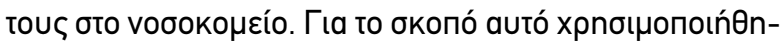

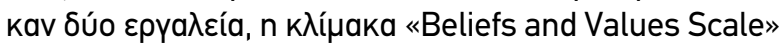

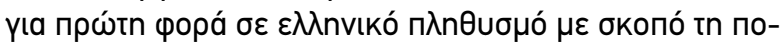

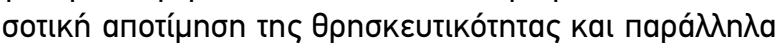

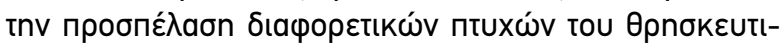

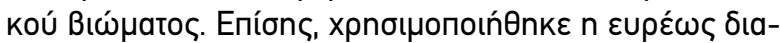
$\delta \varepsilon \delta о \mu \varepsilon ́ v n$ k $\lambda i ́ \mu a k a$ HADS (Hospital Anxiety-Depression

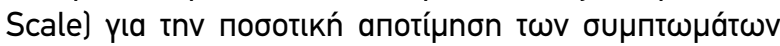
áyxouৎ kaı katá $\theta \lambda ı$

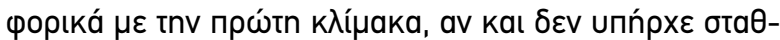

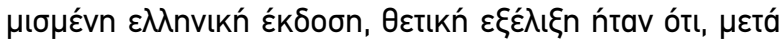

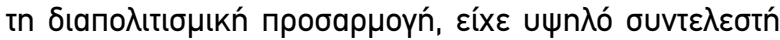




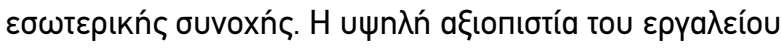

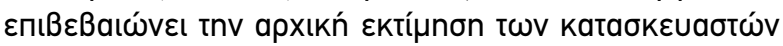

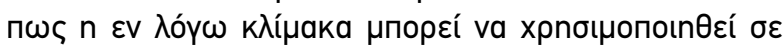

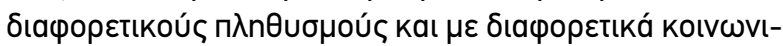

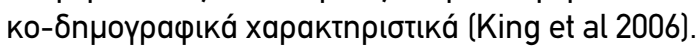

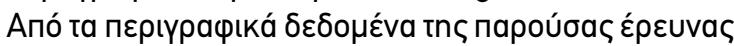

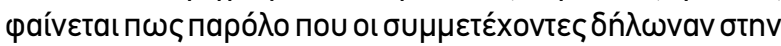

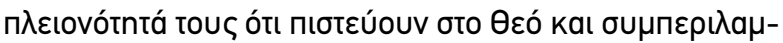

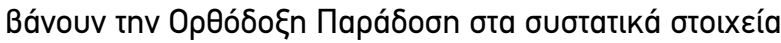

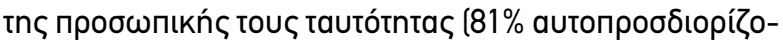

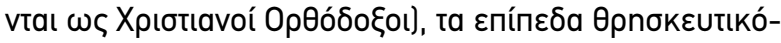

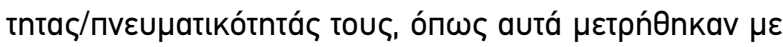

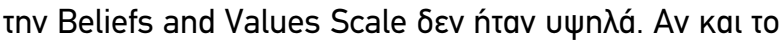

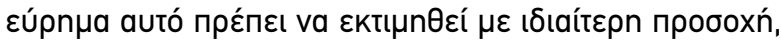

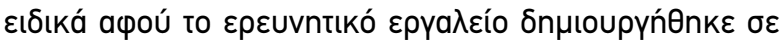

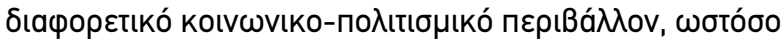

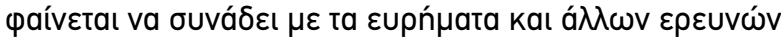

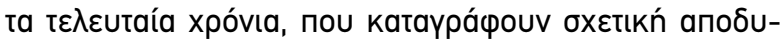

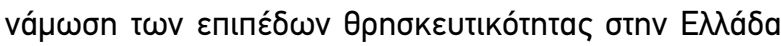

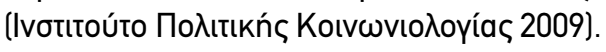

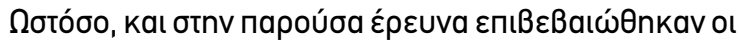

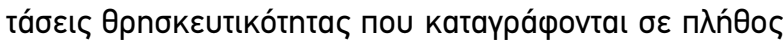

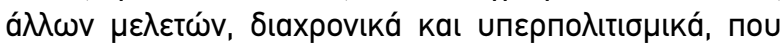

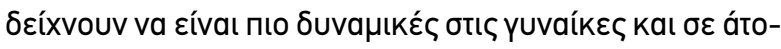

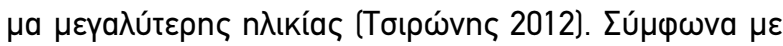

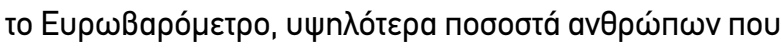

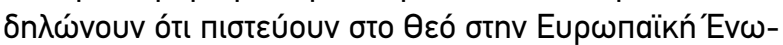

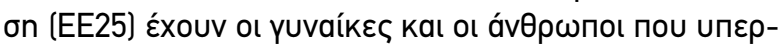

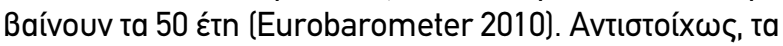

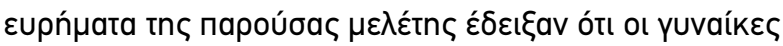

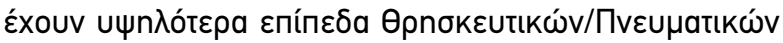

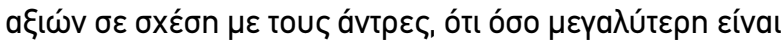

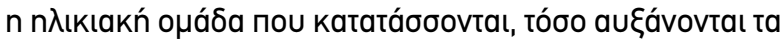

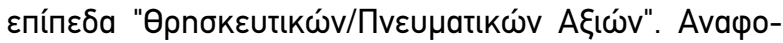

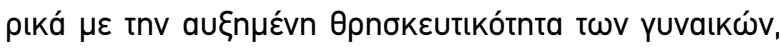

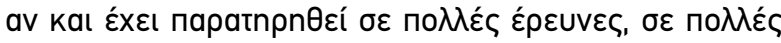

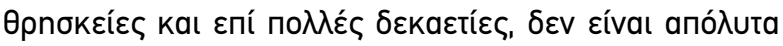

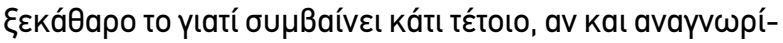

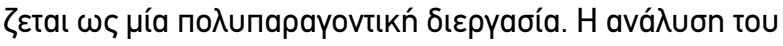

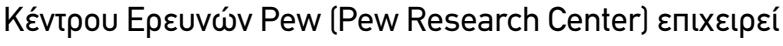

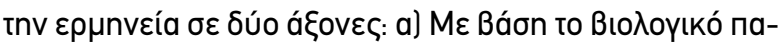

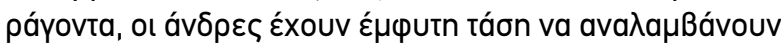

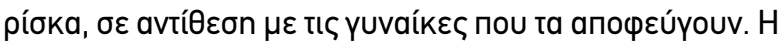

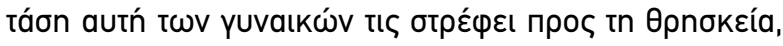

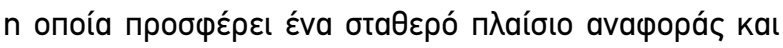

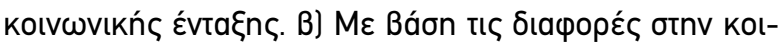

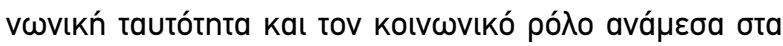

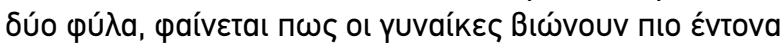

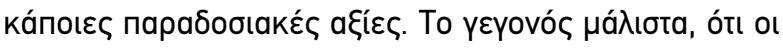

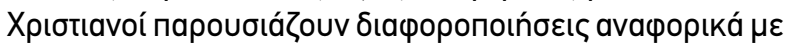

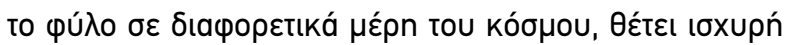

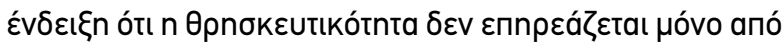

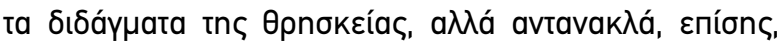

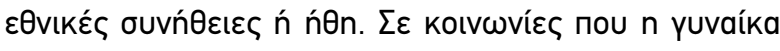

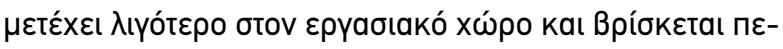

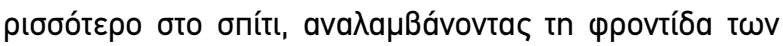

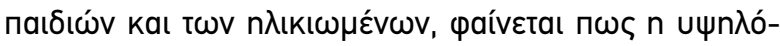

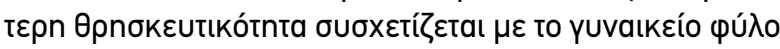
(Pew Research Center 2018).

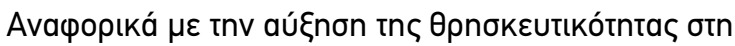

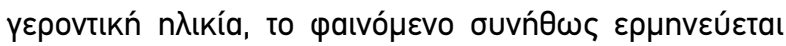

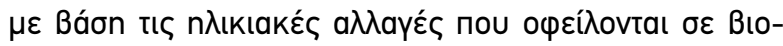

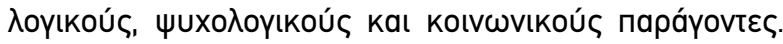

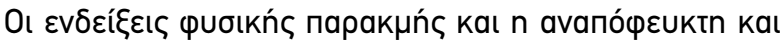

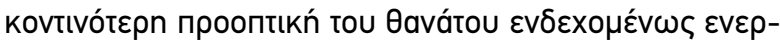

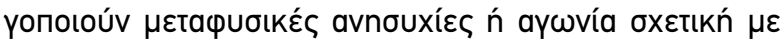

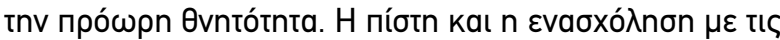

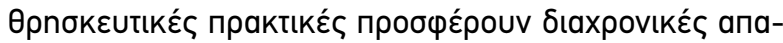

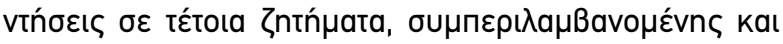

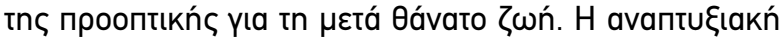

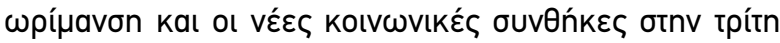

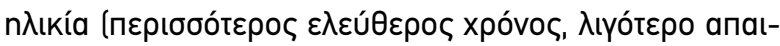

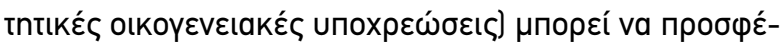

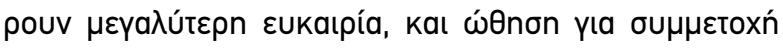

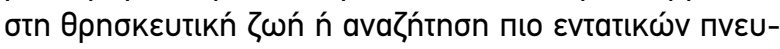

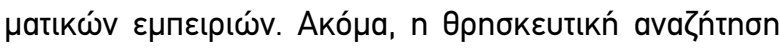

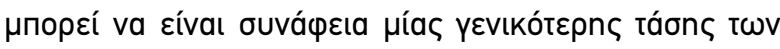

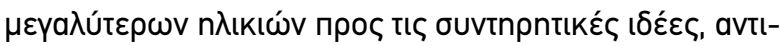

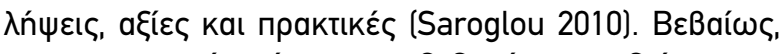

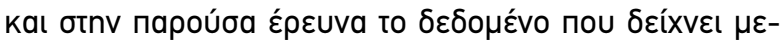

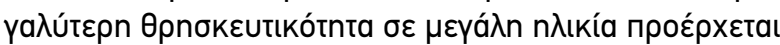

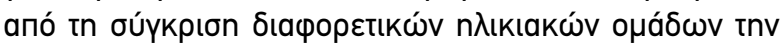

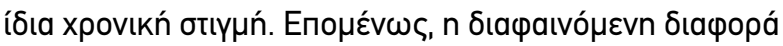

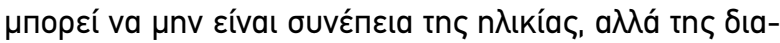

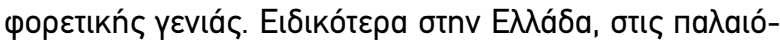

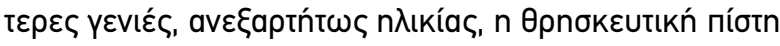

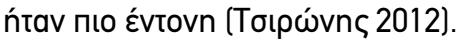

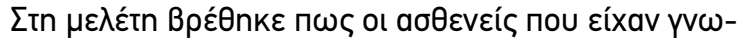

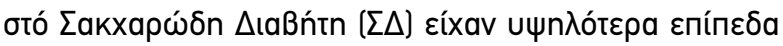

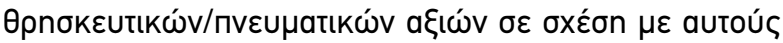

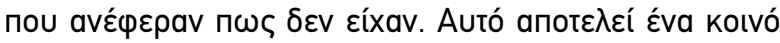

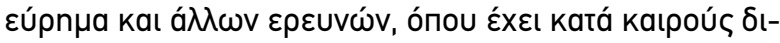

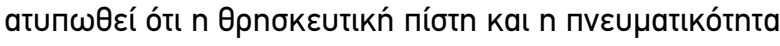

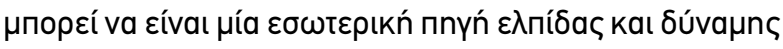

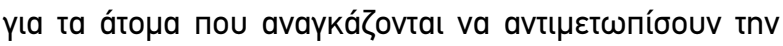

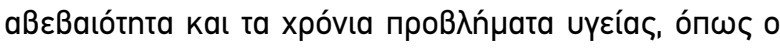

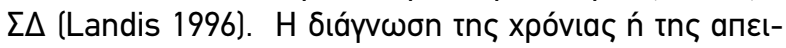

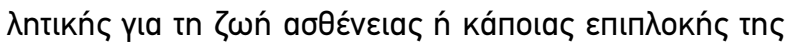

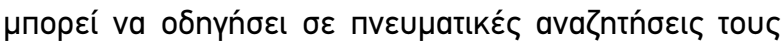

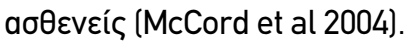

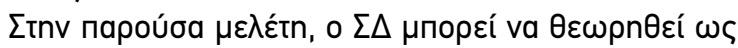

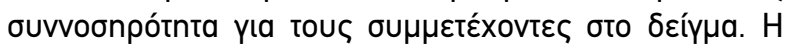

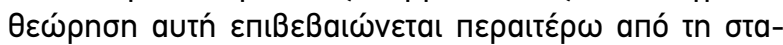

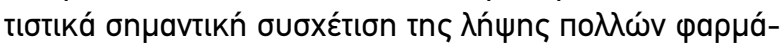
$\kappa \omega v-\omega \varsigma$ ع

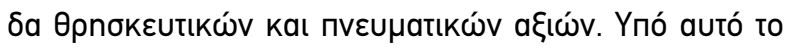




\section{EPEYNHTIKA APOPA}

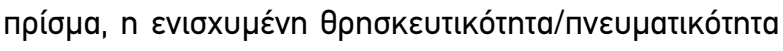

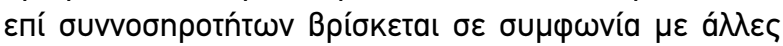

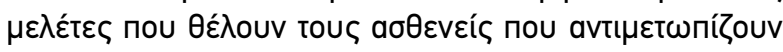

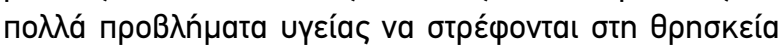

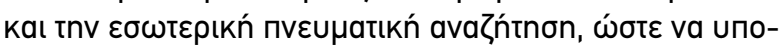

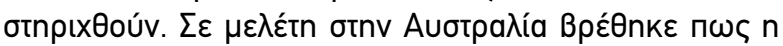

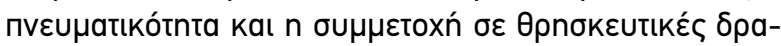

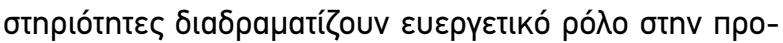

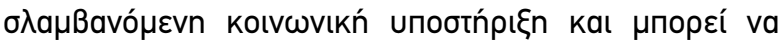

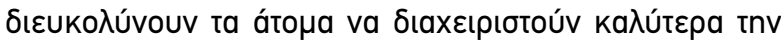

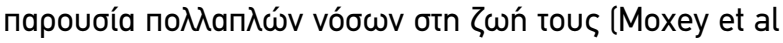
2011).

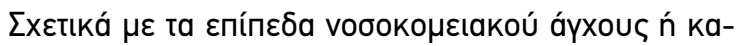

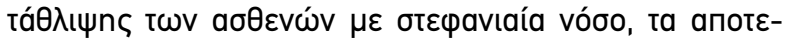

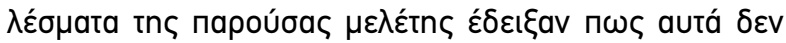

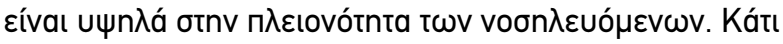

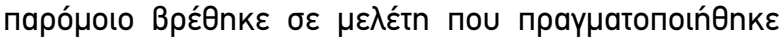

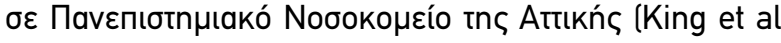

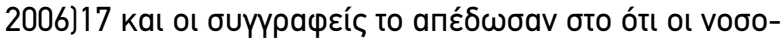

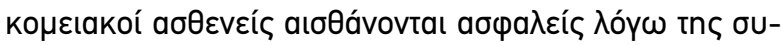

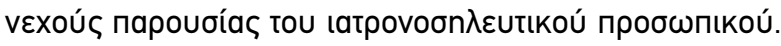

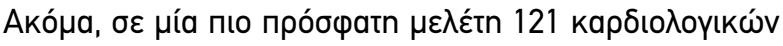

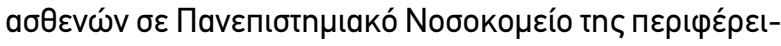

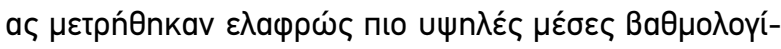

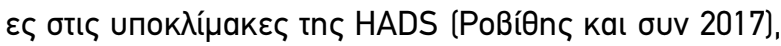

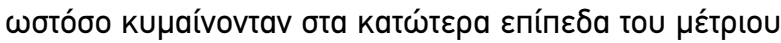

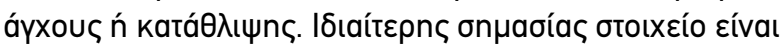

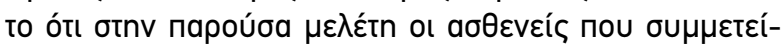

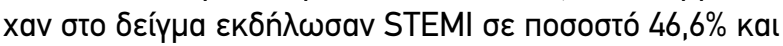

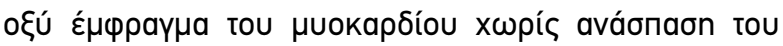

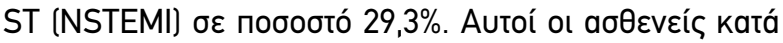

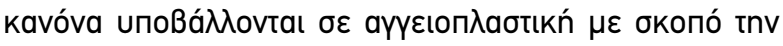

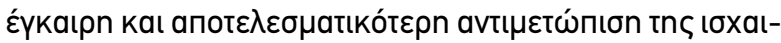

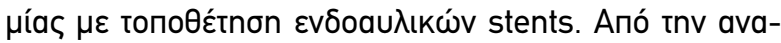

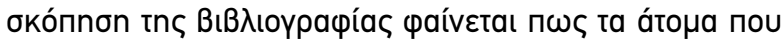

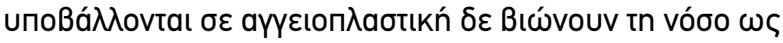

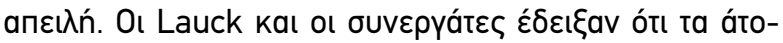

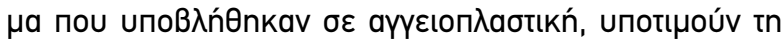

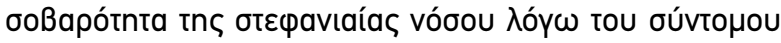

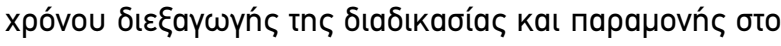

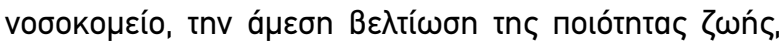

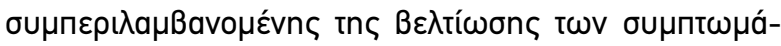

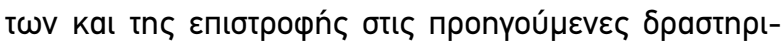

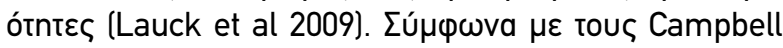

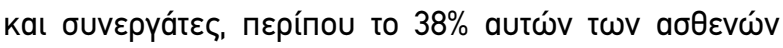

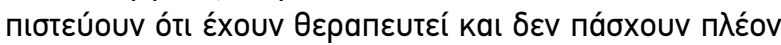

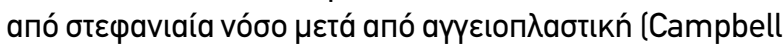
\& Torrance 2005).

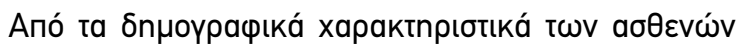

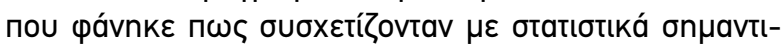

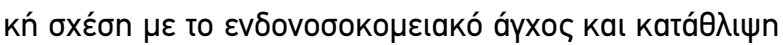

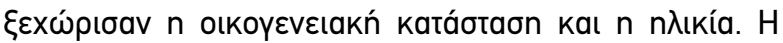

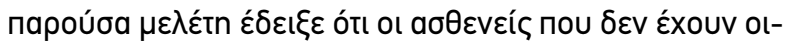

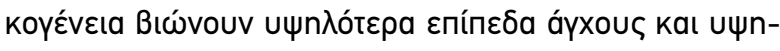

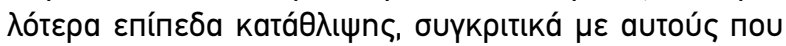

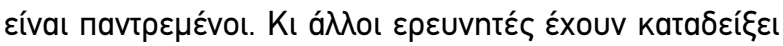

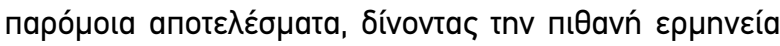

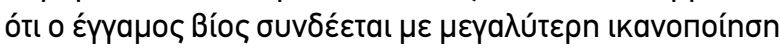

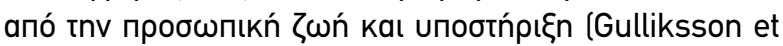

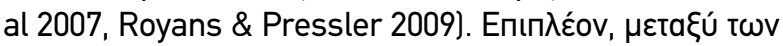

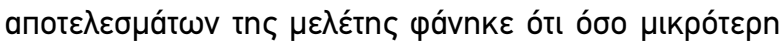

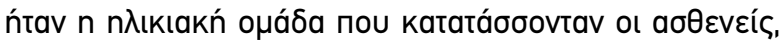

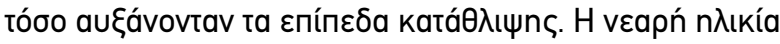

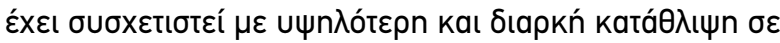

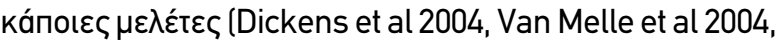

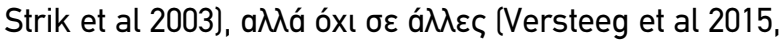

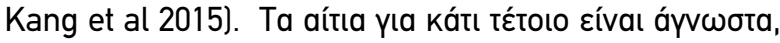

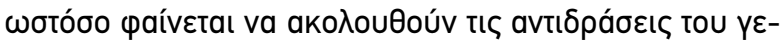

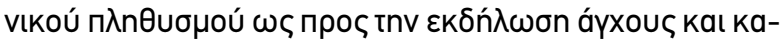

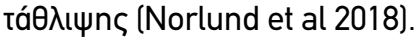

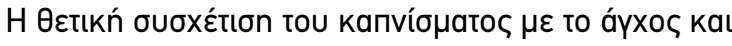
tnv katáӨ

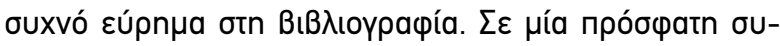

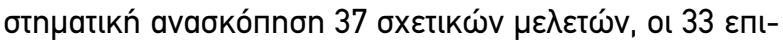

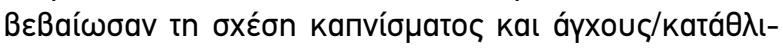

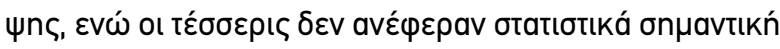

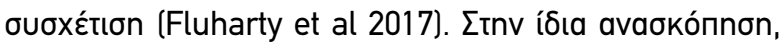

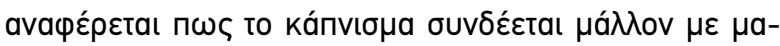

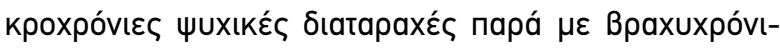

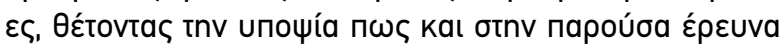

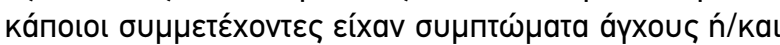

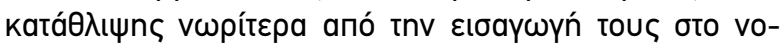

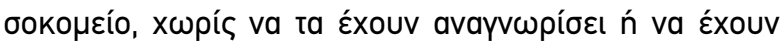

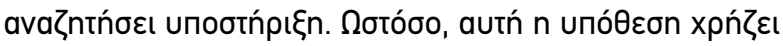

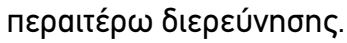

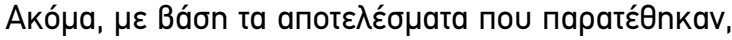

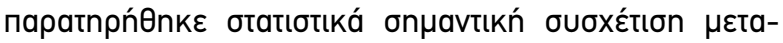

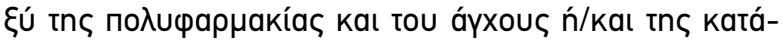

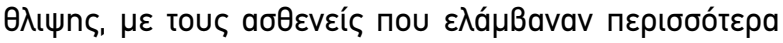

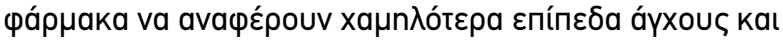

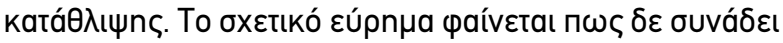

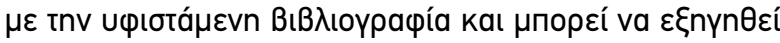

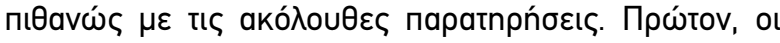

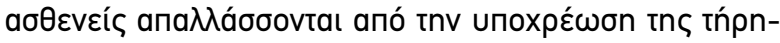

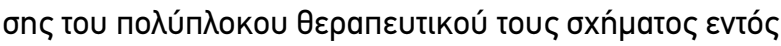

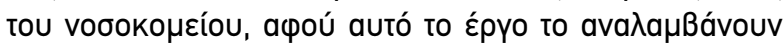

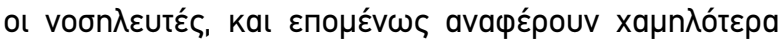

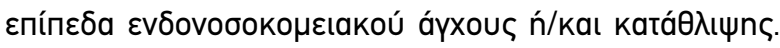

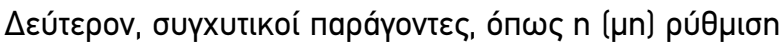

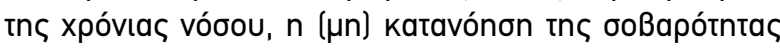

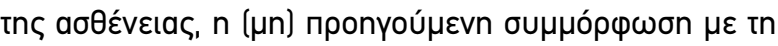

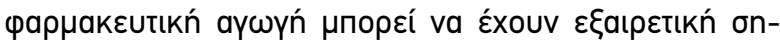

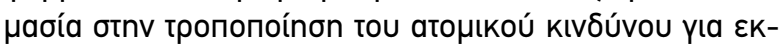

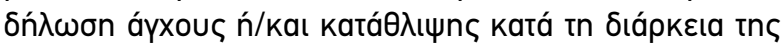

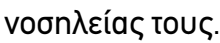

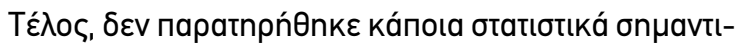




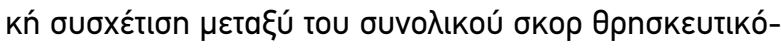

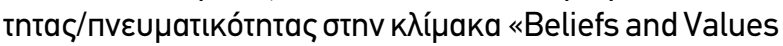

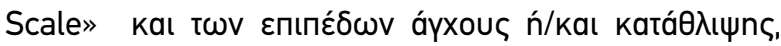

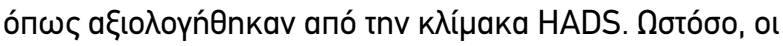

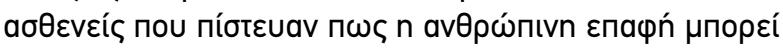

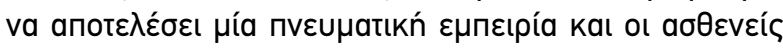

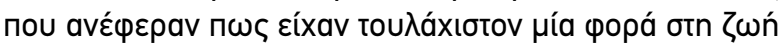

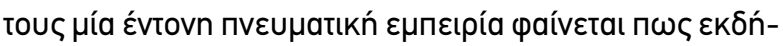

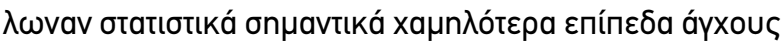

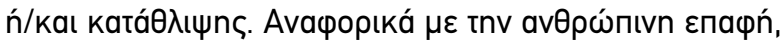

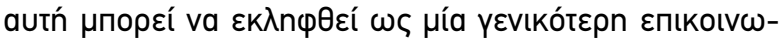

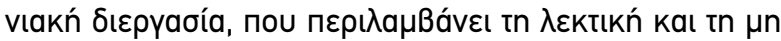

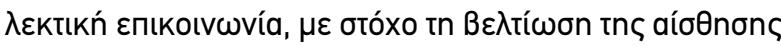

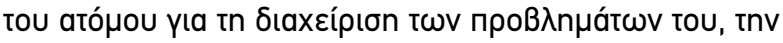

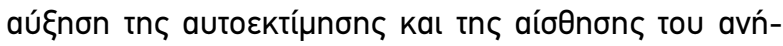

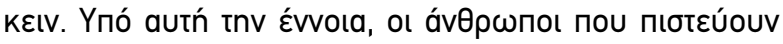

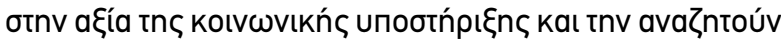

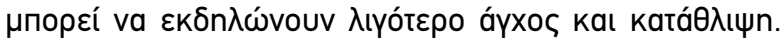

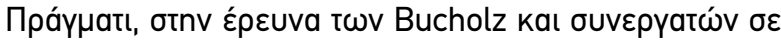

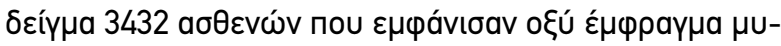

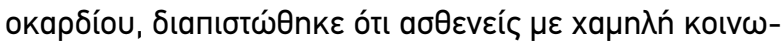

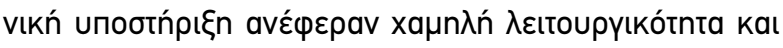

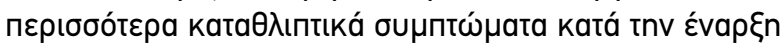

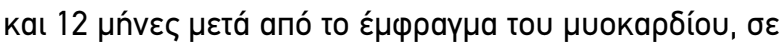

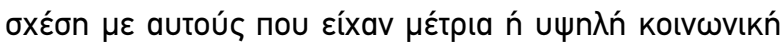

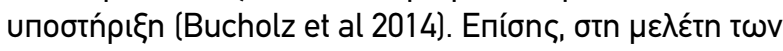

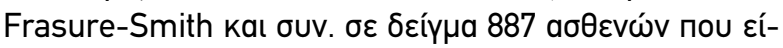
xav uпобтद́ દ́

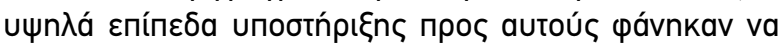

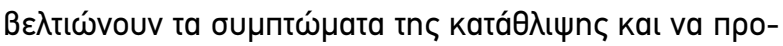

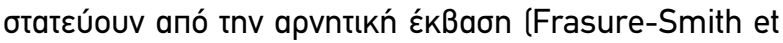

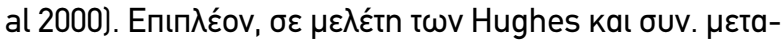

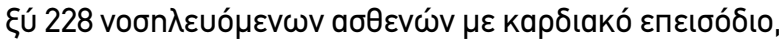

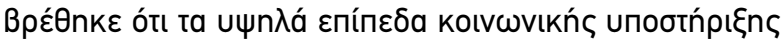

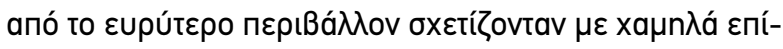

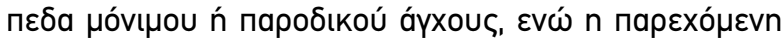

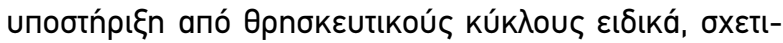

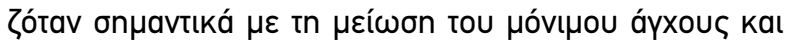

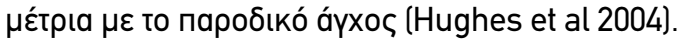

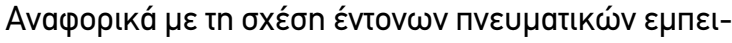

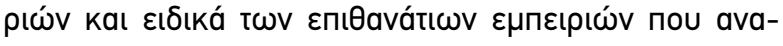

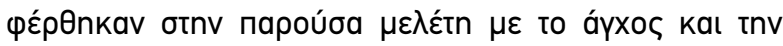

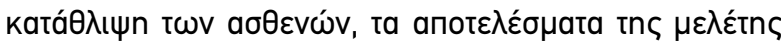

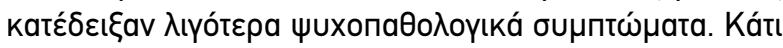

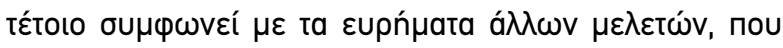

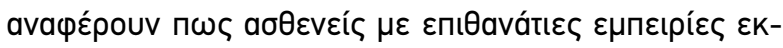

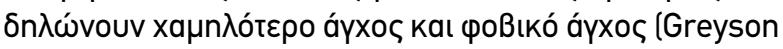

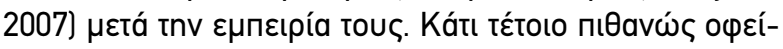

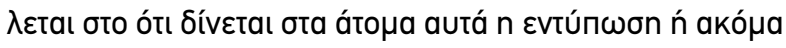

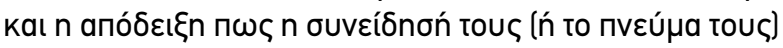

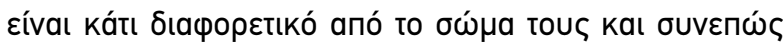

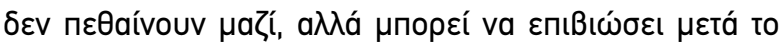
Gávato. H проontıkń tn

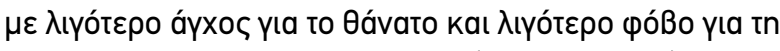

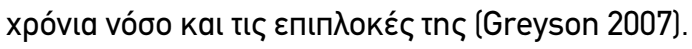

\section{Пврเорเбноі́}

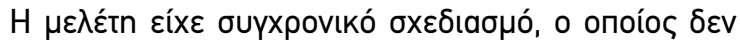

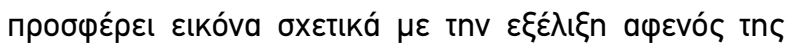

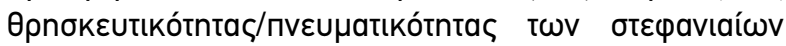

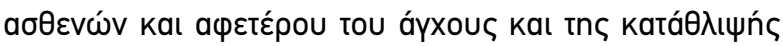

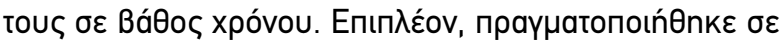

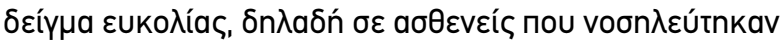

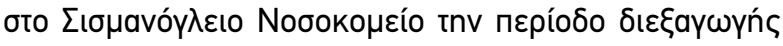

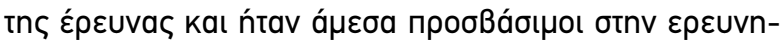

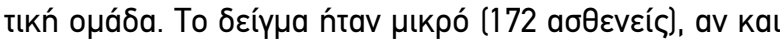

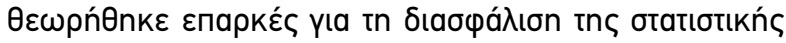

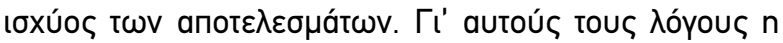

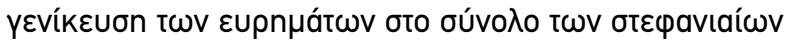

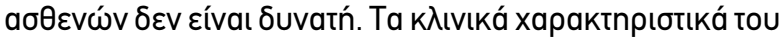

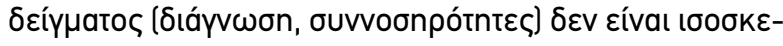

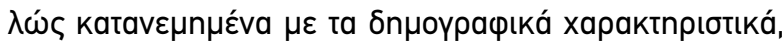

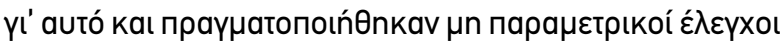

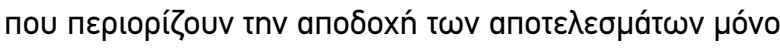

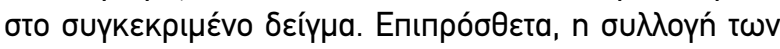

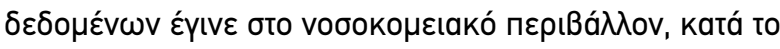

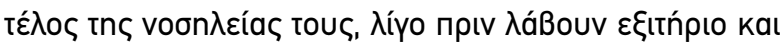

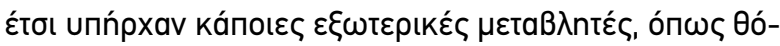

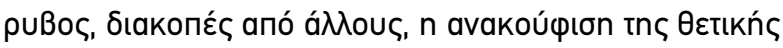

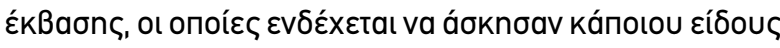

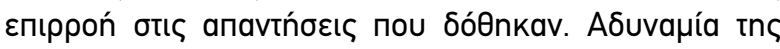

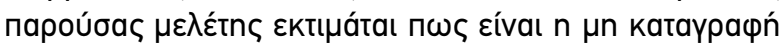

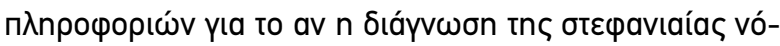

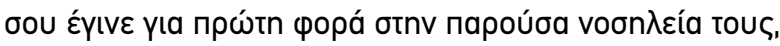

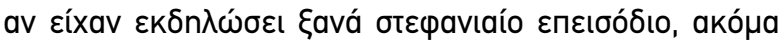

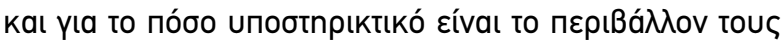

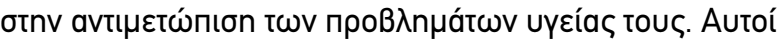

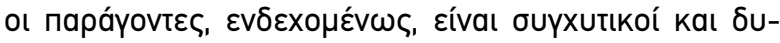

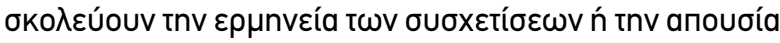
autẃv.

\section{¿YMПEPA¿MATA}

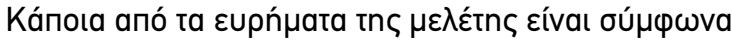

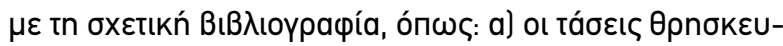

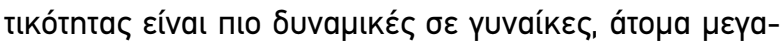

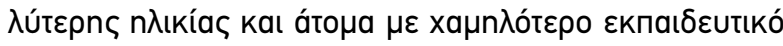

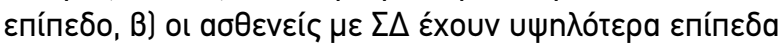

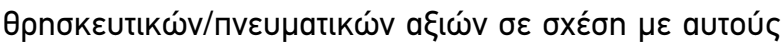

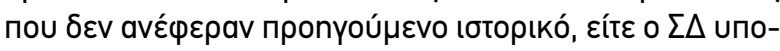

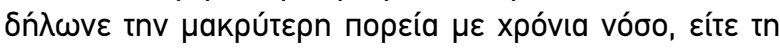

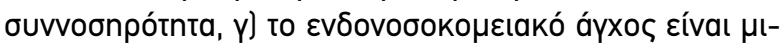

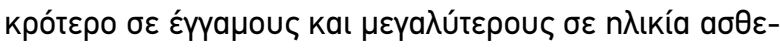

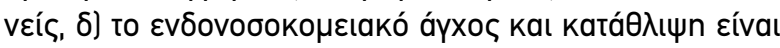

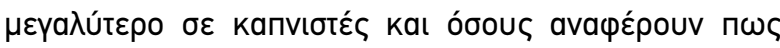

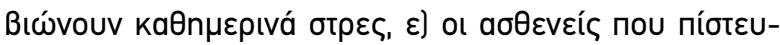

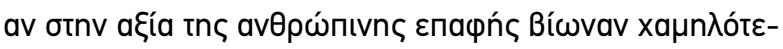




\section{EPEYNHTIKA APOPA}

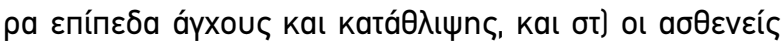

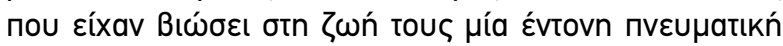

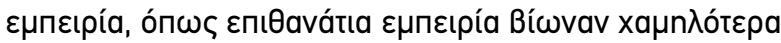

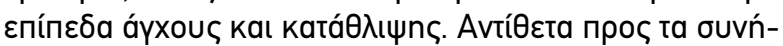

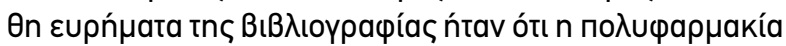

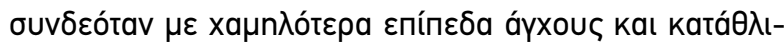

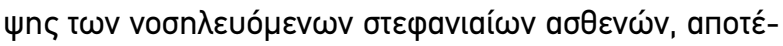

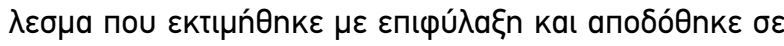

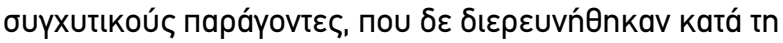

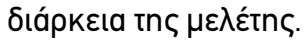

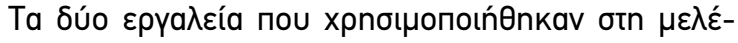
tn (Beliefs and Values Scale \& HADS) paívetaı va avı-

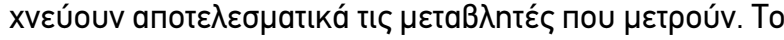

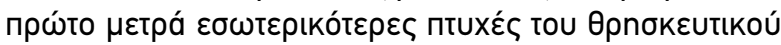

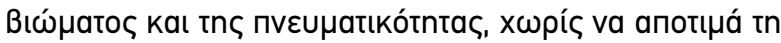

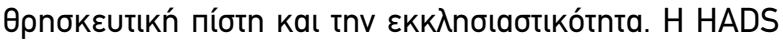

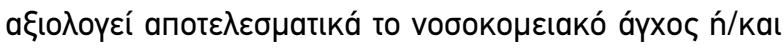

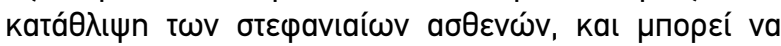

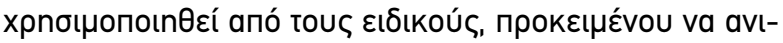

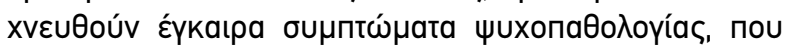

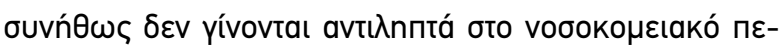

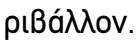

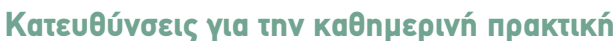

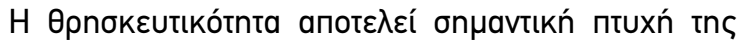

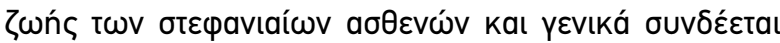

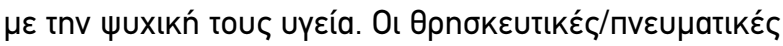

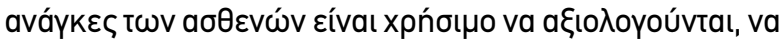

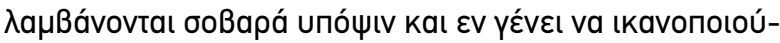

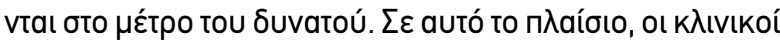

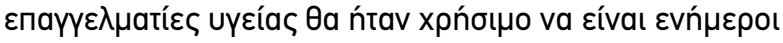

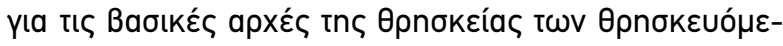

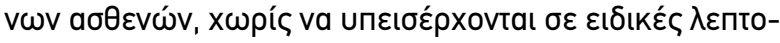

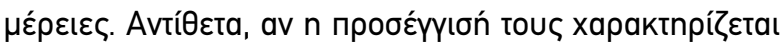

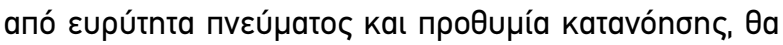

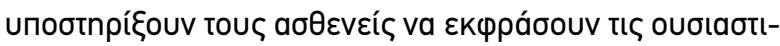

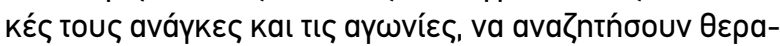

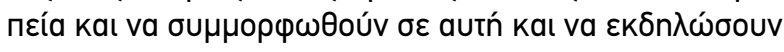

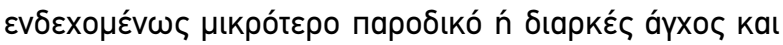
кatá $\theta \lambda ı \Psi n$. 


\section{ВІВАІОГРАФІА}

Aukst-Margetic B. \& Margetic B. (2005). Religiosity and Health Outcomes: a Review of Literature. Coll Antropol 29:365-371.

Bucholz E., Strait K., Dreyer R., Geda M., Spatz E., Bueno H., Lichtman J., D' Onofrio G., Spertus J. \& Krumholz H. (2014). Effect of low perceived social support on health outcomes in young patients with acute myocardial infarction: results from the VIRGO Study. $J$ Am Heart Assoc 3:e00125.

Campbell M. \& Torrance C. (2005). Coronary angioplasty: impact on risk factors and patients' 'understanding of the severity of their condition. Aust J Adv Nurs 22:26-31.

Dickens C., Percival C., McGowan L., Douglas J., Tomenson B., Cotter L., Heagert A. \& Creed F.H. (2004). The risk factors for depression in first myocardial infarction patients. Psychol Med 34:1083-1092.

Ellison C.G. \& George L.K. (1994). Religious involvement, social ties, and social support in a south-eastern community. J Science Study Religion 33:46-61.

Eurobarometer (2010). Social Values, science and technology. $\Delta$ la $\theta \dot{\varepsilon}-$

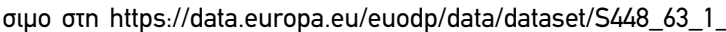

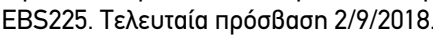

Faul F., Erdfelder E., Lang A.G. \& Buchner A. (2007). G*Power 3 : A flexible statistical power analysis program for the social, behavioral, and biomedical sciences. Behav Res Methods 39:175191

Fitchett G., Rybarczyk B.D., deMarco G.A. \& Nicholas J.J. (1999). The role of religion in medical rehabilitation outcomes: a longitudinal study. Rehabil Psychol 44: 333-353.

Fluharty M., Taylor A.E., Grabski M. \& Munafo M.R. (2017). The association of cigarette smoking with depression and anxiety: a systematic review. Nicotine Tob Res 19:3-13.

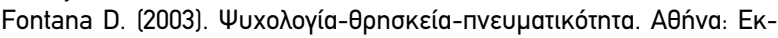

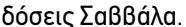

Frasure-Smith N., Lesperance F., Gravel G., Masson A., Juneau M., Talajic M. \& Bourassa M. (2000). Social support, depression and mortality during the forst year after myocardial infarction. Circulation 101:191-192.

Ginting H., Naring G., Kwankkebon L. \& Becker E. (2015). Spirituality and negative emotions in individuals with coronary heart disease. J Cardiovasc Nurs 30:537-545.

Greyson B. (2007). Near-death experiences: clinical implications. Rev. Psiq. Clín 34: 49-57.

Gulliksson M., Burell G., Lundin L., Toss H. \& Svardsudd K. (2007). Psychosocial factors during the first year after a coronary heart disease event in cases and referents. Secondary Prevention in Uppsala Primary Health Care Project (SUPRIM). BMC Cardiovasc Disord 7:36.

Hughes J., Tomlinson A., Blumenthal J., Davidson J., Sketch M. \& Watkins L. (2004). Social support and religiosity as coping strategies for anxiety in hospitalized cardiac patients. Ann Behav Med 28:179-188.

Ironson G., Solomon G.F., Balbin E.G., O' Cleirigh C., George A., Kumar M., Larson D. \& Woods T.E. (2002). The Ironson-Woods spirituality/religiousness index is associated with long survival, health behaviors, less distress and low cortisol in people with HIV/AIDS. Ann Behav Med 24: 34-48.

Jenkinson C., Wright L. \& Coulter A. (1994). Criterion validity and reliability of the SF-36 in a population sample. Qual Life Res 3:712.

Kang H., Stewart R., Bae K.Y., Kim S.W., Shin I.S., Hong Y.J., Ahn Y., Jeong M.H., Yoon J.S. \& Kim J.M. (2015). Predictors of depressive disorders following acute coronary syndrome: results from K-DEPACS and EsDEPACS. J Affect Disord 181:1-8.

Kennedy J.E., Abbott R.A. \& Rosenberg B.S. (2002). Changes in spirituality and well-being in a retreat program for cardiac patients. Altern Ther Health Med 8:64-73

King M., Jones L., Barnes K., Low J., Walker C., Wilkinson S., Mason C., Sutherland J. \& Tookman A. (2006). Measuring spiritual belief: development and standardization of a Beliefs and Values Scale Psychol Med 36:417-425.

Koenig H.G., McCullogh M. \& Larson D.B. (2001). Handbook of religion and health. Oxford, England: Oxford University Press.

Landis B.J. (1996). Uncertainty, spiritual well-being, and psychological adjustment to chronic illness. Issues Ment Health Nurs 17:217231

Lauck S., Johnson J.L. \& Ratner P.A. (2009). Self-care behavior and factors associated with patients outcomes following same-day discharge percutaneous coronary intervention. Eur J Cardiovasc Nurs 8:190-199.

McCord G., Gilchrist V.J., Grossman S.D., King B.D., McCormick K.W., Oprandi A.M., Schrop S.L., Selious B.A., Smucker D.O., Weldy D.L., Amorn M., Carter M.A., Deak A.J., Hefzy H. \& Srivastara M. (2004). Discussing spirituality with patients: a rational and ethical approach. Ann Fam Med 2:356-361.

McLean C.D., Susi B., Phifer N., Schulz L. \& Franco M (2003). Patient preference for physician and practice of spirituality. $J$ Gen Intern Med 18(1): 38-43.

Meador K.G., Koenig H.G., Hughes D.C., Blazer D.G., Turnball J. \& George L.K. (1992). Religious affiliation and major depression. Hosp Community Psychiatry 1992, 43:1204-1208.

Moxey A., McEvoy M., Bowe S. \& Attia J. (2011). Spirituality, religion, social support and health among older Australian adults. Australas J Ageing 30:82-88.

Newberg A.B. \& Lee B.Y. (2006). The relationship between religion and health. In McNamara P. (ed): Where God and Science meet? The Psychology of religious experience, London: Praenger Publications.

Norlund F., Lissaker C., Wallert J., Held C. \& Olsson E.M. (2018). Factors associated with emotional distress in patients with myocardial infarction: results from the SWEDEHEART registry. Eur J Prev Cardiol 25:910-290.

Pew Research Center (2018). The gender gap in religion around the

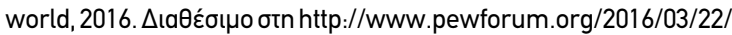

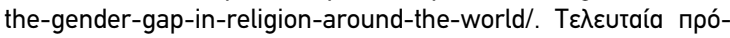

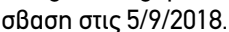

Royans L.M. \& Pressler S.J. (2009). Depressive symptoms and heart failure: examining the sociodemographic variables. Clin Nurse Spec 23: 138-144

Saroglou V. (2010). Late adulthood and religiosity. In P. Vassileiadis (ed). Great Orthodox Christian Encyclopedia AӨńva: ¿трatnүıќ์

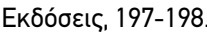

Strik J.J., Denollet J., Lousberg R. \& Honig A. (2003). Comparing symptoms of depression and anxiety as predictors of cardiac events and increased health care consumption after myocardial infarction. J Am Coll of Cardiol 42:1801-1807.

Van Melle J.P., De Jonge P., Spijkerman T.A. (2004). Prognostic association of depression following myocardial infarction with mortality and cardiovascular events: a meta-analysis. Psychosom Med 66:814-822.

Versteeg H., Roest A.M. \& Denollet J. (2015). Persistent and fluctuating anxiety levels in the 18 months following acute myocardial infarction: the role of personality. Gen Hosp Psychiatry 37:1-6.

Weber S. \& Pargament K. (2014). The role of religion and spirituality in mental health. Curr Opin Psychiatry 27:358-363.

Zigmond A.S. \& Snaith R.P. (1983). The Hospital Anxiety and Depression Scale. Acta Psychiatr Scand 67:361-370.

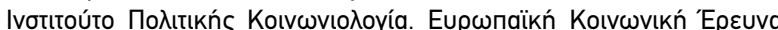

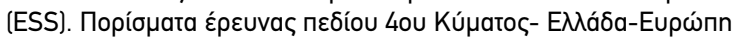




\section{EPEYNHTIKA APOPA}

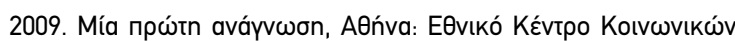

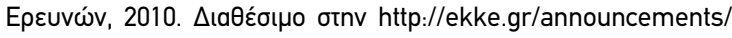

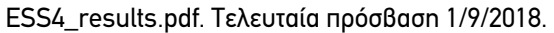

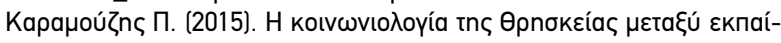

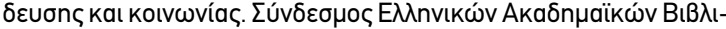
oӨnkẃv, AӨńva.

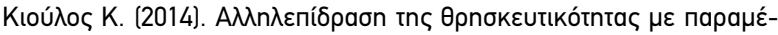

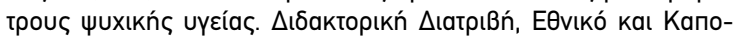

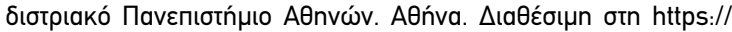

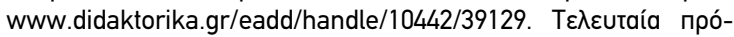

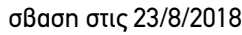

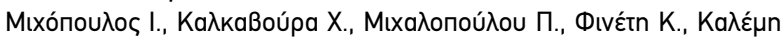

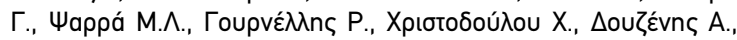

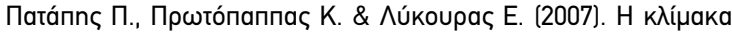

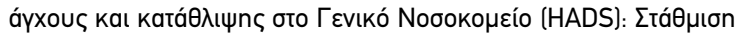

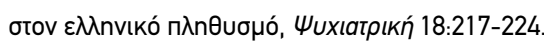

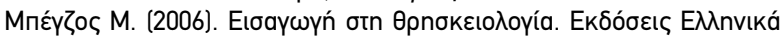

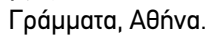

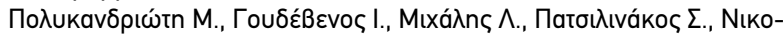

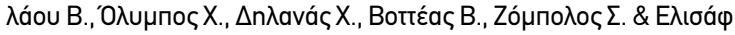

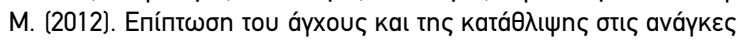

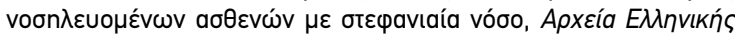
latpıкńs 29:33-43.

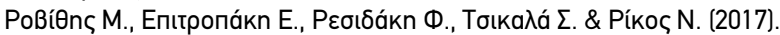

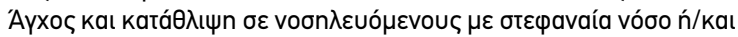

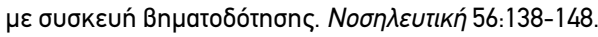

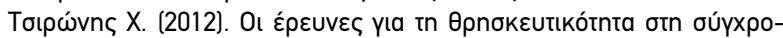

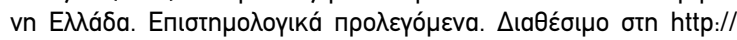

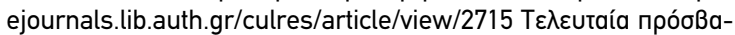
on $8 / 6 / 2018$. 


\title{
The effect of religiosity of patients with Coronary Artery Disease on in-hospital anxiety and depression
}

Maria Goudinoudi1', Vasiliki Karra², Konstantinos Ekmetzogloư ${ }^{3}$, Anna Korombeli4, Evangelia Kouskouni ${ }^{5}$ 1. RN, MSc, Coronary Intensive Care Unit, General Hospital Sismanoglio-Amalia Fleming (Sismanoglio Hospital) 2. RN, MSc, MHCM, PhD, Vice Director of Nursing Department General Hospital Sismanoglio-Amalia Fleming (Sismanoglio Hospital) 3. MD, PhD, Lecturer, Medical School, European University of Cyprus

4. RN, MSc, PhD, Special Educational Personnel, Faculty of Nursing, National and Kapodistrian University of Athens

5. Professor Emeritus of Biopathology, National and Kapodistrian University of Athens

\begin{abstract}
Introduction: Religious faith and spirituality often seem to affect physical and psychological functioning of patients with chronic diseases.

Aim: To investigate the relationship between religiosity/spirituality of coronary patients with anxiety and depression which may be experienced during their hospitalization.

Methods: A cross-sectional study was conducted involving 172 patients (23 female and 149 male) with coronary artery disease, who were hospitalized in a general hospital of Attica region with an acute coronary syndrome diagnosis. HADS scale was used for the investigation of hospital anxiety and depression and the Belief and Values Scale was used to evaluate the patients' religious faith and spirituality.

Results: Moderate levels of religiosity/spirituality and no to moderate levels of anxiety and depression were found in coronary patients. No statistically significant correlations were found between the HADS subscales and the Beliefs and Values Scale $(p>0.001)$, although individuals who have experienced strong spiritual experiences, experienced less anxiety and depression in hospital. There were found statistically significant correlations ( $p<0.001$ ) with gender, religious beliefs, patients' age with Belief and Values Scale. There were also statistically significant correlations $(p<0.001)$ of HADS subscales with family status, age, smoking habits, stress and medication.

Conclusion: Highly religious coronary patients are less likely to experience anxiety and depression during hospitalization, though confounders seem to interfere and make it difficult to interpret this relationship. Further research is required in order to explore the impact of religiosity on psychological distress of hospitalized coronary patients.
\end{abstract}

Key words: Anxiety, coronary disease, depression, hospital, religiosity, spirituality

Citation: Goudinoudi M., Karra V., Ekmetzoglou K., Korombeli A. \& Kouskouni E. (2020). The effect of religiosity of patients with Coronary Artery Disease on ih-hospital anxiety and depression. Hellenic Journal of Nursing Science 13(3): 43-59, https://doi.org/10.24283/ hjns.202036 